\title{
Navigation Technology for Exploration of Glacier Ice With Maneuverable Melting Probes
}

\author{
J. Kowalski ${ }^{\mathrm{a}, \mathrm{i}, *}$, P. Linder ${ }^{\mathrm{b}, \mathrm{c}, *}$, S. Zierke ${ }^{\mathrm{c}, *}$, B. von Wulfen ${ }^{\mathrm{d}, *}$, J. Clemens ${ }^{\mathrm{e}, *}$, \\ K. Konstantinidis ${ }^{\mathrm{f}, *}$, G. Ameres ${ }^{\mathrm{g}}$, R. Hoffmann ${ }^{\mathrm{h}, *}$, J. Mikucki ${ }^{\mathrm{j}}$, S. Tulaczyk ${ }^{\mathrm{k}}$, \\ O. Funke ${ }^{\mathrm{l}}$, D. Blandfort ${ }^{\mathrm{a}}$, C. Espe ${ }^{\mathrm{a}}$, M. Feldmann ${ }^{\mathrm{a}}$, G. Francke ${ }^{\mathrm{a}}$, S. Hiecker ${ }^{\mathrm{a}}$, \\ E. Plescher ${ }^{\mathrm{a}}$, S. Schöngarth ${ }^{\mathrm{a}}$, K. Schüller ${ }^{\mathrm{a}}$, B. Dachwald ${ }^{\mathrm{a}}$, I. Digel ${ }^{\mathrm{b}}$, \\ G. Artmann ${ }^{\mathrm{b}}$, D. Eliseev ${ }^{\mathrm{c}}$, D. Heinen ${ }^{\mathrm{c}}$, F. Scholz ${ }^{\mathrm{c}}$, C. Wiebusch ${ }^{\mathrm{c}}$, S. Macht ${ }^{\mathrm{d}}$, \\ U. Bestmann $^{\mathrm{d}}$, T. Reineking ${ }^{\mathrm{e}}$, C. Zetzsche ${ }^{\mathrm{e}}$, K. Schille ${ }^{\mathrm{e}}$ R. Förstner ${ }^{\mathrm{f}}$, \\ H. Niedermeierg , A. Szumskig , B. Eissfellerg,*, U. Naumann ${ }^{\mathrm{h}}$, K. Helbing ${ }^{\mathrm{h}}$ \\ ${ }^{a}$ Faculty of Aerospace Engineering, FH Aachen University of Applied Sciences, Germany \\ ${ }^{b}$ Institute of Bioengineering, FH Aachen University of Applied Sciences, Germany \\ ${ }^{c}$ III. Physikalisches Institut, RWTH Aachen University, Germany \\ ${ }^{d}$ Institute of Flight Guidance, Technische Universität Braunschweig, Germany \\ ${ }^{e}$ Cognitive Neuroinformatics, University of Bremen, Germany \\ ${ }^{f}$ Institute of Space Technology and Space Applications LRT 9.1, Universität der \\ Bundeswehr München, Germany \\ ${ }^{g}$ Institute of Space Technology and Space Applications LRT 9.3, Universität der \\ Bundeswehr München, Germany \\ ${ }^{h}$ Department of Physics, Bergische Universität Wuppertal, Germany \\ ${ }^{i}$ AICES, RWTH Aachen University, Germany \\ ${ }^{j}$ Department of Microbiology, University of Tennessee Knoxville, USA \\ ${ }^{k}$ Department of Earth and Planetary Science, University of California Santa Cruz, USA \\ ${ }^{l}$ DLR Space Administration, Germany
}

\begin{abstract}
The Saturnian moon Enceladus with its extensive water bodies underneath a thick ice sheet cover is a potential candidate for extraterrestrial life. Direct ex-

\footnotetext{
*Corresponding authors (authorship contributions are detailed in section 6)

Email addresses: kowalski@aices.rwth-aachen.de (J. Kowalski), linder@fh-aachen.de (P.Linder), simon.zierke@rwth-aachen.de (S.Zierke), b.vonwulfen@tu-bs.de (B. von Wulfen), clemens@uni-bremen.de (J. Clemens), k.konstantinidis@unibw.de (K. Konstantinidis), rhoffman@uni-wuppertal.de (R. Hoffmann), bernd.eissfeller@unibw.de (B. Eissfeller)
}

Preprint submitted to Cold Regions Science and Technology

November 8, 2015

(C) 2015. This manuscript version is made available under the Elsevier user license http://www.elsevier.com/open-access/userlicense/1.0/ 
ploration of such extraterrestrial aquatic ecosystems requires advanced access and sampling technologies with a high level of autonomy. A new technological approach has been developed as part of the collaborative research project Enceladus Explorer (EnEx). The concept is based upon a minimally invasive melting probe called the IceMole. The force-regulated, heater-controlled IceMole is able to travel along a curved trajectory as well as upwards. Hence, it allows maneuvers which may be necessary for obstacle avoidance or target selection. Maneuverability, however, necessitates a sophisticated on-board navigation system capable of autonomous operations. The development of such a navigational system has been the focal part of the EnEx project. The original IceMole has been further developed to include relative positioning based on in-ice attitude determination, acoustic positioning, ultrasonic obstacle and target detection integrated through a high-level sensor fusion. This paper describes the EnEx technology and discusses implications for an actual extraterrestrial mission concept.

Keywords: minimally-invasive melting technology, in-ice dead reckoning, acoustic positioning, acoustic reconnaissance, multi-sensor fusion, clean sampling 
1

2

3

4

5

6

7

\section{Introduction}

\subsection{Subglacial Liquid Water Reservoirs on Enceladus}

It is now widely accepted that the presence of liquid water on planets and moons of our Solar System bears some potential for the development of extraterrestrial life. One astrobiologically relevant candidate is the Saturnian moon Enceladus [1, 2, 3]. At Enceladus' South Polar Terrain, ice grains that contain organic compounds are released and ejected into space through fractures, which are informally called Tiger Stripes. Those fractures are located along four parallel valleys, which are about $200-250 \mathrm{~m}$ deep and about $50-100 \mathrm{~m}$ wide $[4,5]$. Measurements of the Cassini spacecraft imply that those jets or curtains of particles originate from a subsurface salt-water reservoir in contact with rock and that they are transported through the $30-40 \mathrm{~km}$ thick ice layer via cracks [6, 7]. Within the Tiger Stripe fractures, temperatures up to $167 \mathrm{~K}$ have been observed [8], significantly warmer than the expected $68 \mathrm{~K}$ for this region. On a smaller scale, modeled temperatures of hot spots approach $200 \mathrm{~K}$ [4]. This has most convincingly been attributed to latent heat carried by water from the subglacial ocean, and deposited via water vapor along the walls of the cracks. These locations are considered as promising regions for the search for extraterrestrial life forms.

\subsection{Direct Subglacial Exploration}

Currently, our ability to detect and characterize living organisms on distant bodies remains a search for biosignatures, such as polynucleotides, cellular structures, and expected metabolic products (e.g. $\mathrm{CH}_{4}$ ), in samples observed and probed from space or returned to Earth [5]. Such biosignatures 
are extremely difficult to characterize in a reliable way, and much more so once they have been exposed to the vacuum and the radiation of space. To carry out a scientific investigation, we hence seek to directly access and sample subglacial aquatic ecosystems by targeting water-filled cracks at a moderate depth in which liquid water still communicates with large water aquifers at deeper depth, e.g. a subglacial ocean. Direct exploration requires advanced accessing and sampling technologies. Such a technology is provided by the IceMole, a maneuverable, subsurface icecraft for exploration and in-situ analysis within ice $[9,10]$.

\subsection{Joint Research Collaboration Enceladus Explorer}

The IceMole combines drilling with an ice screw to control the contact force exerted on the ice with a differential heating system at the melting head and the main body of the probe. This enables the probe to melt along a curved trajectory or even upwards. Such melting maneuvers, however, imply an on-board navigation system capable of monitoring position and attitude and ambient environment of the probe at any point in time. This also is a main prerequisite for autonomous operations. Within the joint research collaboration Enceladus Explorer (EnEx), a sophisticated in-ice navigation system has been developed and integrated with the original IceMole probe into the EnEx system. Furthermore, an integrated clean access protocol has been developed and tested that allows contamination-free deployment and clean sample collection. In this publication, we provide a detailed introduction to the EnEx system, which consists of the IceMole melting probe enhanced by a navigational payload and a surface control module. In particular, we will describe 
1. the general carrier and sampling system,

2. the relative positioning based on attitude determination,

3. the acoustic based absolute positioning,

4. the ultrasonic fore-field exploration technology,

5. a sensor fusion approach to consolidate the acquired data,

and present results from laboratory and field tests both in the European Alps, and in the Dry Valleys, Antarctica.

\section{EnEx System}

\subsection{General Design}

The complete EnEx system consists of the EnEx-IceMole as well as various surface based devices (figure 1). The EnEx-IceMole is a third generation IceMole $^{1}$. Its concept is based upon earlier work on the first and second generation IceMole probes $[9,10]$. In its current version the probe has a cross section of $0.15 \mathrm{~m} \times 0.15 \mathrm{~m}$ and a length of $2.0 \mathrm{~m}$, including a $6 \mathrm{~cm}$ ice screw at the front of the melting head. It's mass is approximately $60 \mathrm{~kg}$. Conceptually, the probe consists of two parts:

1. The EnEx carrier system includes the melting head with the drive and heating systems and a general body structure containing main circuit board, communications and power control.

2. Modular payload assembled in custom boxes (dead reckoning box, acoustic box, sample container).

\footnotetext{
${ }^{1}$ In this publication reference to the IceMole or 'the probe' refers to the EnEx-IceMole generation.
} 


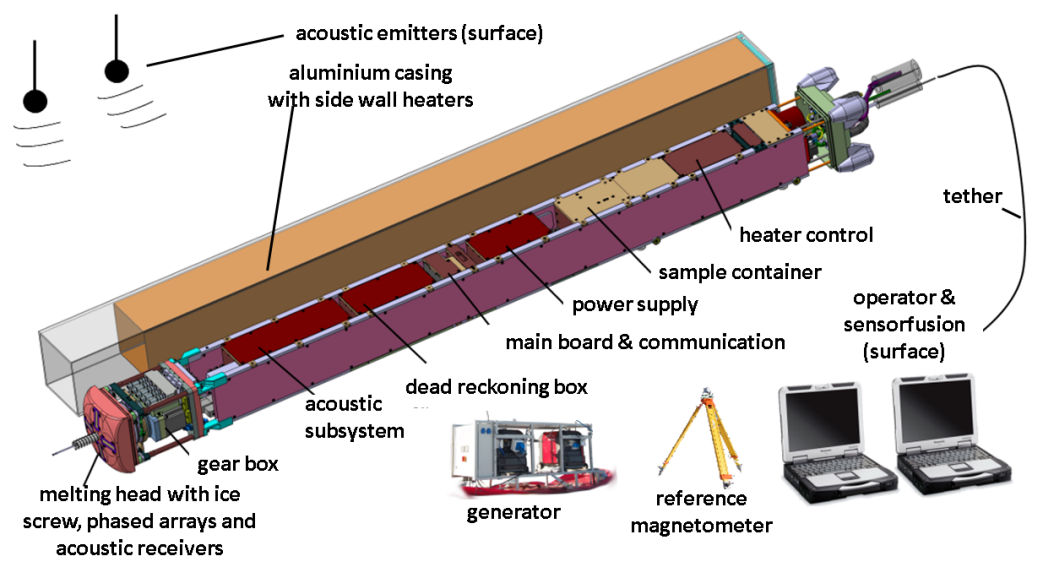

Figure 1: The EnEx system consists of the IceMole upgraded with navigational instrumentation and integrated with a surface power and control system. The surface component includes a ruggedized laptop for data integration and communication, a generator, a reference magnetometer and acoustic reference points. The IceMole probe is connected to the surface by a power cable which plugs into the generator and data cables communicating with the computer as well as with a water sampling tube. The IceMole includes the melting head with the drive and heating systems and a general body structure containing main circuit board, communications and power control. It contains a modular payload assembled in custom boxes (dead reckoning box, acoustic box, sample container), which can easily be pulled out for servicing.

71 Within the IceMole, parts of the carrier system are connected via a CAN bus, whereas the modular payload systems communicate over ethernet with the main circuit board and the surface control systems. The detailed design of the copper melting head can be seen in figure 2. Figure 3 shows the interior of the melting head. The convex head is equipped with two sets of eight heating cartridges of $200 \mathrm{~W}$ and $160 \mathrm{~W}$ which add to a maximum forward melting power of $2.88 \mathrm{~kW}$. A standard mountaineering ice screw is mounted at the 


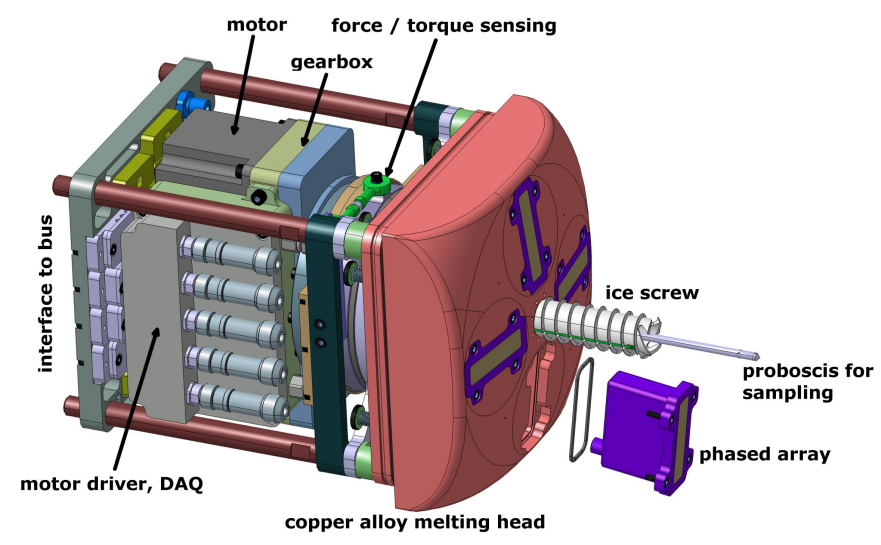

Figure 2: CAD image of the highly integrated melting head. Two motors, (one being redundant to minimize risk), a gearbox and a customized data acquisition system operates the force-regulated ice screw. In order to take the water sample, a hollow proboscis can be deployed through the inside of the screw. Four phased piezo arrays are integrated into the melting head, one being dismantled in this schematic. The arrays are aligned crosswise around the screw leaning $7.2 \mathrm{deg}$ to the outside.

center of the melting head. The drive system allows for a maximum power of $25 \mathrm{~W}$. It can be configured to maintain a contact force between 0 and 2000 N. Most types of glacier ice are not strong enough for the highest contact forces to be applied, such that realistic values range from $300 \mathrm{~N}$ to $800 \mathrm{~N}$. In the nominal melting scenario the operator sets the heating configuration and a maximum contact force. The resulting melting velocity is internally regulated by a feedback control. Side wall heaters with a linear power gradient are integrated along the hull $\left(0,5-2 \mathrm{~W} / \mathrm{cm}^{2}\right)$. Tests showed that the back plate has to be heated as well to assure retraction capability in curved and twisted channels. The back melting plate has a power output of up to $0.8 \mathrm{~kW}$. The proboscis for water sample acquisition is deployed from the hollow ice 
screw (figure 2). The picture furthermore shows four ultrasonic phased arrays and indicates the position of acoustic receivers. Both are components of the navigation suite and will be detailed in section 3.2 on acoustic in-ice positioning.

\subsection{Operating Interface and Surface Connection}

The IceMole has a threefold permanent connection to the surface station. The three lines are all bundled inside a protecting spring wire reinforced polyurethane hose. It has a total length of $40 \mathrm{~m}$. The bundled tether is uncoiled from a cable drum at the surface. The single lines are:

1. A data line connection to the surface computer system: Full-duplex mode communication between the IceMole main computer, the payload systems and the computer at the surface station is realized via ethernet (1000BASE-T). A RS422 time syncronisation line for the acoustic positioning is also provided (section 3.2).

2. A power cable with $3 \times 2,5 \mathrm{~mm}$ cross section (eq. AWG13) connecting the probe to the generators $(2 \mathrm{x} 3 \mathrm{kVA}, 230 \mathrm{~V}$ line voltage): This cable is additionally used for communication via a power line modem. Hence, a redundant communication method is available to minimize the risk of losing the probe.

3. A fluid tube connection, which operates in two different modes: (1) upward pumping of meltwater or any other liquid present in the melting channel and (2) downward pumping of a sterilizing hydrogen peroxide solution. 
The IceMole is operated from within a custom-built EnEx Graphical User Interface (EnEx GUI) operated on the surface computer. It yields real-time data and control access to the heating system, the ice screw and the internal sensors (temperature, pressure, etc.). The EnEx run control software displays a summary status of the IceMole and is responsible for the surveillance and control of the EnEx GUI and the navigation subsystems. Communication with the subsystems is handled by the User Datagram Protocol (UDP). Besides its surveillance tasks, the EnEx run control initiates automatic stops of the IceMole to guarantee undisturbed data acquisition for the navigation subsystems. These stops are referred to as measurement stops. First, a stop command is sent from the EnEx run control to the EnEx GUI. Once the EnEx GUI receives information about the upcoming measurement stop, it turns off the heaters, stops the drive and tightens the screw, to bring the IceMole into a stationary state. Then, the measurement sequence starts. Afterwards, a data summary is displayed in the EnEx run control software to verify data quality. Finally, the data is passed onto the high-level sensor fusion module (section 3.4). Results of the sensor fusion are displayed in the EnEx navigation GUI and provide operator support for strategic decisions during mission execution.

\subsection{Melting Motion of the IceMole}

A heating power of the forward melting system of $2.88 \mathrm{~kW}$ has been determined in the design phase as the heat flux necessary to maintain a melting velocity of $1 \mathrm{~m} / \mathrm{h}$ for the probe cross-section of 0.15 by $0.15 \mathrm{~m}^{2}$. Contingencies account for conductive losses and the fact that the melting channel will be somewhat larger in area than the IceMole itself. This approach 


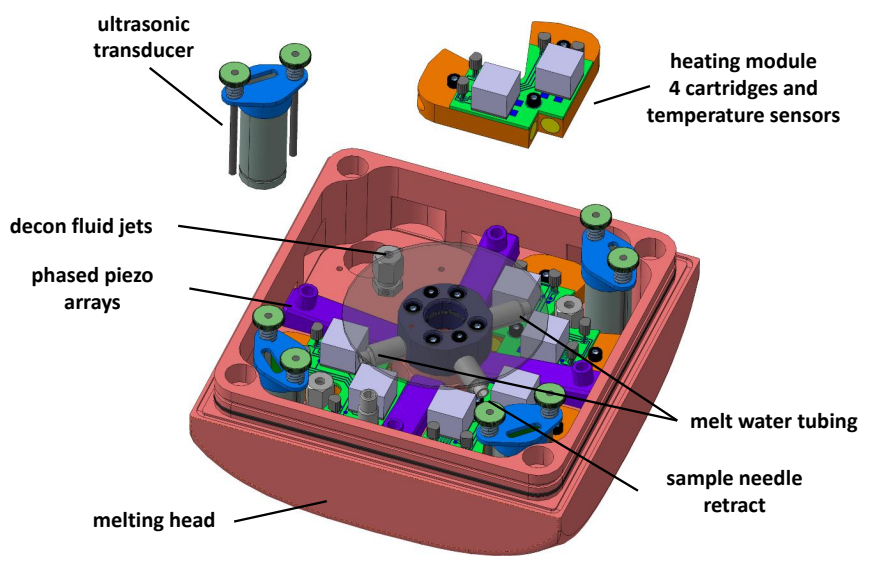

Figure 3: This image shows the backside of the melting head. The four acoustic receivers are located in the corners, the phased piezo arrays are aligned crosswise around the centered screw. Close to the center the tubing for sample retraction and the warm water emergency system can be seen. Four fluid releases are distributed across the melting head to assure optimal spreading of the sterilizing fluid.

is following [11] and assumes a standard atmospheric pressure of 1 bar as well as an ice temperature of $-17^{\circ} \mathrm{C}$, such as expected at Taylor Glacier in Antarctica. This approach is well established for conventional melting probes [12]. In order to analyze geometry and surface material influence on the melting performance however, one has to go beyond a bulk energy balance consideration and set up a spatially realistic close-contact melting model. Close-contact melting [13] can be observed whenever a heat source, in our case the melting head, is pressed against a phase-change material, in our case glacier ice. For the IceMole, this contact force results from probe weight and the pulling force exerted by the ice screw. Melting in the vicinity of the probe induces its motion into the glacier. The probe is separated from ice by a very thin liquid film (figure 4). Characteristic scales in the 


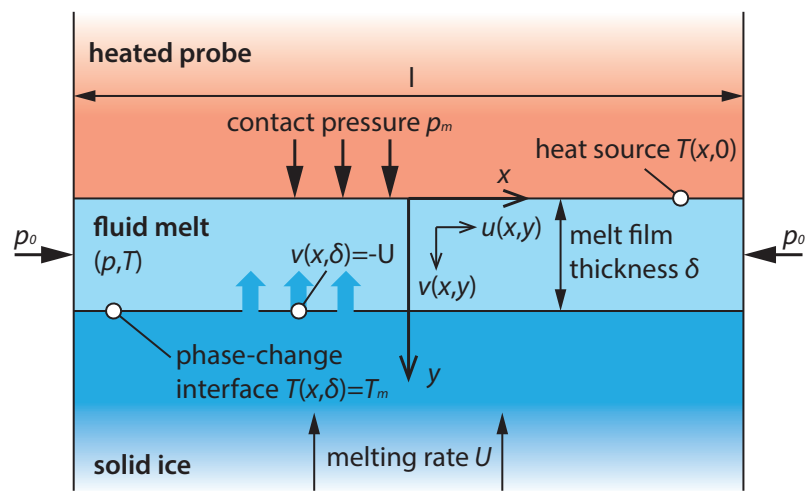

Figure 4: Sketch of the microscale liquid film in front of the probe which is generated between the heated probe and the ice. The width of the melting head is indicated by $l$. The phase-change is assumed to occur along a clearly defined interface (located at $\delta$ ) which is at melting temperature $\mathrm{T}_{m}$. Tangential and normal fluid velocity are given by $\mathrm{u}$ and $\mathrm{v}$. For a given heater and ice screw configuration, the probe is assumed to descent into the ice at constant melting velocity U. Pressure and temperature of the melt are denoted by $\mathrm{p}$ and T.

system are the thin-film parameter (aspect ratio of melt film thickness and width of the probe), the Reynolds Number and the Peclet Number. For terrestrial conditions, both thin-film parameter, and Reynolds Number will be very small, and provide a supporting rationale for the solution commonly chosen in the literature [13]. Namely, melting velocity, melt film thickness and temperature profile are calculated from incompressible lubrication theory coupled to an open boundary heat equation, known as a Stefan problem. The position of the phase-change interface is given implicitly by requiring a local heat balance at the interface. The mathematical system is numerically solved in two and three dimensions [14]. Reflecting the heater control, the model is implemented with a constant temperature at the probe's melting head. 
For moderate contact forces this boundary condition realistically models the thermal situation of the IceMole. For a high contact force (higher than the maximum force of $800 \mathrm{~N}$ usually observed in field test), a constant temperature at the melting head implies heat fluxes exceeding the available power of the IceMole $(2.88 \mathrm{~kW})$ and this boundary condition would not be appropriate. Results are in good agreement to [15], who state that a paraboloid melting head is optimal in terms of efficiency. For curvilinear melting we observed good agreement between simulation results and laboratory experiments [16] Set up for the IceMole system design, simulations indicate a non-uniform temperature distribution and the development of cold ice cones in front of the acoustic phased arrays (figure 2). In accordance to that, melting velocities observed in field tests have been lower than predicted by the classical theory. These observations can be attributed to the lower thermal conductivity of the sensor's epoxy surface as compared to copper. A copper casing of the phased arrays improved the situation, as has been observed in both experiment and simulations. Though this modification in principle assures the IceMole's melting capability on terrestrial glacier ice, the application of front-viewing sensors to much colder ice, e.g. on Enceladus, implies a revision of the current melting head design.

\subsection{Clean Sampling System}

Two of the field tests have been conducted in Antartica. Hence, the EnEx system had to be in compliance with the Antarctic Treaty that provides requirements for operating instrumentation in the pristine Antarctic ecosystem of Blood Falls [17]. Thus, the sampling and instrument operation has to be conducted in a manner that prevents contamination of the test sites. 
Cleanliness is also a key aspect for any extraterrestrial mission to avoid contamination of a potential extraterrestrial habitat and to maintain integrity of any detected signs of life. Therefore special procedures for cleaning and sterilizing of the contacting hardware and bioload monitoring during assembly, before launch and during the mission were taken into account. The clean access strategy is introduced in greater detail in [10]. In this paper we restrict ourselves to a description of the technological aspects of the clean sampling system.

\subsubsection{In-situ Decontamination and Sample Quality Assurance}

The IceMole has an integrated Clean Access and Sampling Subsystem (CASS). In-situ sterilizing is conducted before the probe reaches its target. For this step, any remaining melt water in the melting channel is pumped out through an internal pumping system, which is optionally supported by additional booster pumps towed behind the probe (figure 1). Next, $3 \%$ hydrogen peroxide is injected into the melting channel through four orifices at the melting head, as well as through the sampling proboscis (figure 2). Injection pressure is powered by an inert gas (argon or nitrogen) that is connected to a pressure vessel filled with $100 \mathrm{dL}$ of $3 \% \mathrm{H}_{2} \mathrm{O}_{2}$. Both the inert gas cylinder and the tank containing the $3 \% \mathrm{H}_{2} \mathrm{O}_{2}$ solution are located at the surface station and connected to the IceMole by the fluid tube connection (see 2.1). The operating pressure for the decontamination system is approximately 10 bar. After an appropriate reaction time (approximately $15 \mathrm{~min}$ ) the decontamination fluid is no longer traceable and the probe is clean. Now the probe can approach the target and tap the water filled crevasse with the proboscis deployed by a pneumatic system. A first sample is pumped to 
the surface for testing. This strategy assures that no remaining $\mathrm{H}_{2} \mathrm{O}_{2}$ solution is in either tubes or melting channel. Once cleanliness has been confirmed, pristine brine samples are collected into two internal, sterile, gas-tight sample bags ( $5 \mathrm{dl}$ each). The sampling bags are located in two separate gas tight boxes and connected to the proboscis through a series of valves. The bags are filled by producing an under-pressure in the sample bag boxes (approximate filling time is $180 \mathrm{sec}$ ). Optionally, the brine can be pumped to the surface directly through a hose and sampled there by the booster pumps. Although the latter seems to be the simpler approach, care has to be taken as the sample alters its biochemical structure immediately after being exposed to oxygen in the air.

\subsubsection{Emergency Rescue System}

Clean access not only means to avoid contamination of the environment and assurance of a clean sample, but also to minimize the risk of losing the probe in the melting channel since the probe itself represents potential contamination. In addition to its main function, the CASS provides an emergency rescue system for the IceMole in case of a complete system failure and loss of communications. In this case, the pressure vessel of the decontamination system on the surface will be filled up with clean hot water. The hot water will be forced through the fluid tube to the orifices at the melting head and will release the ice screw from the ice, allowing the probe to be extracted through the melting channel and returned to the surface. 


\section{In-ice Navigation}

Within the EnEx project a sophisticated navigation solution has been developed and integrated into the probe's navigation suite. It consists of four modules:

1. Dead Reckoning (DR) based on inertial and magnetic attitude determination,

2. an Acoustic Positioning System (APS) that yields the absolute position based on trilateration of acoustic signals,

3. an Acoustic Reconnaissance System (ARS) based on sonography for fore-field exploration, and

4. a high-level Sensor Fusion (SF) unit to integrate the acquired DR, APS, and ARS data.

The four modules are described in detail in the following subsections.

\subsection{DR for Relative Position and Attitude Determination}

Dead Reckoning (DR) means to determine the position of the melting probe relative to a given starting point based upon its attitude evolution and information on the distance traveled. The distance traveled is determined by counting the motor-steps and correct them by gear ratio and ice screw thread pitch. The attitude of the IceMole is determined by an Attitude and Heading Reference System (AHRS) which relies on gyroscope readings of an Inertial Measurement Unit (IMU). In a second step the AHRS attitude solution is fused with the magnetic heading determined from differential magnetometry to limit drift and improve accuracy. Both AHRS, and the 
differential magnetometer system are described in the following subsections. Further details can also be found in [18].

\subsubsection{Attitude and Heading Reference System}

Long mission times of up to $100 \mathrm{~h}$ and more and slow translational and rotational motion are major challenges for inertial sensors. High angular accuracy is needed to achieve a position accuracy of $1 \mathrm{~m}$ for a distance of $60 \mathrm{~m}$. Priority is to control the system drift, while the update rate is of secondary importance. Usage in polar regions limits the accuracy of the independent determination of the north direction. The chosen fiber-optic gyroscopes (FOG) type inertial measurement unit (IMU) Northrop Grumman LN-200 matches both, the performance requirements and additional geometric constraints in the IceMole's DR box (figure 1). Data and power conversion is tailored to the IMU specifications. Integration angles, sensor biases and noise parameters for the Kalman filter have been determined from rate table tests and Allan variance analysis [19]. The AHRS algorithm carries out a quaternion based strap-down attitude calculation. It is corrected by a Kalman filter, which uses gravity aiding by the accelerometers as well as heading updates from the differential magnetometer system. Important steps of the algorithm are:

1. Initial Alignment: The initial alignment of the IMU with respect to a north-oriented, local navigation frame is a crucial step in order to keep initial errors small. It is performed by measurements of the Earth rotation vector $\omega_{\mathbf{E}}$ and the gravity vector $\mathbf{g}$. At first the east-axis is defined as the cross product of $\mathbf{g}$ and $\omega_{\mathbf{E}}$, and secondly the north-axis is defined as the cross-product of east-axis and $\mathbf{g}$. The alignment procedure 


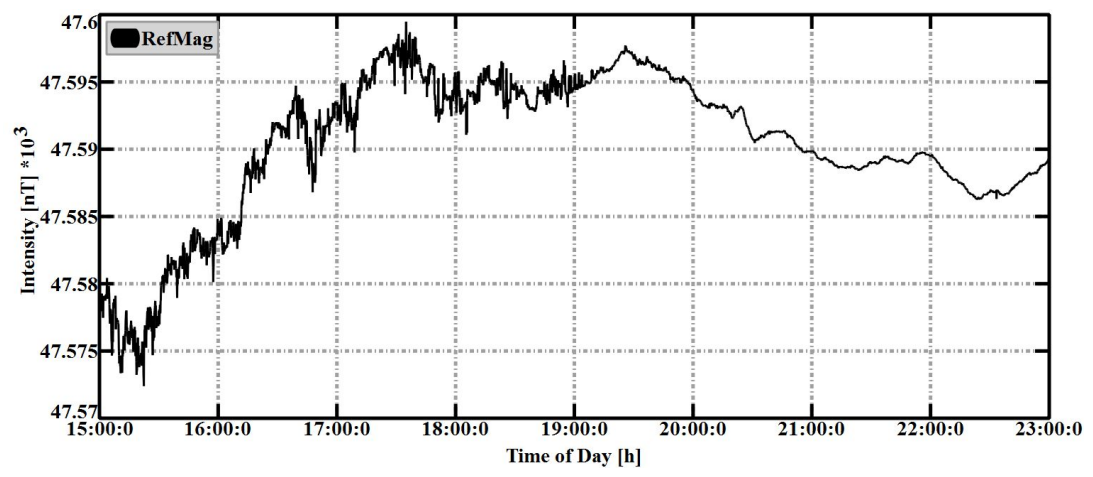

Figure 5: Diurnal magnetic field intensity measured in the Dry Valleys, Antarctica. Longterm variations in total intensity are shown with overlaid short-term variations such as noise. The change of noise level in the intensity plot before 19:00 correlates with the sunset.

takes at least four minutes. Its duration could be further shortened by additional consideration of the magnetic heading and fine alignment filtering.

2. Attitude propagation by gyroscopic readings: Since the IceMole is able to operate with steep pitch angles and vertically, the attitude propagation algorithm is based on a singularity-free quaternion implementation. Differential change of the quaternion is determined using the last available attitude, the fraction of the earth rotation in the update interval and the rotation rate measured by the gyroscopes [20].

3. Error state prediction through a Kalman filter: Despite initial calibration, small sensor errors cannot be totally avoided and there will be 
an offset between true and calculated attitude of the IceMole. The AHRS is very sensitive to errors of the gyroscopic readings, such that a small initial sensor bias will produce large attitude errors eventually. Hence, further error correction is necessary. Updates from the magnetometer system and the APS can be used to correct residual attitude errors, while the prerequisite is that the AHRS filter is robust enough to continue operation with imprecise initial attitude estimation. In this project, a filter based on the Psi-angle error model [21, 22] was adopted to the melting case. The filter uses a nine state design, including attitude and the gyroscope errors biases and scale factor errors. The system noise model is based on the LN-200 gyroscopes random walk, rate random walk and bias stability. The observation model noise uses the accelerometer noise, as well as an estimate of the magnetometer heading accuracy. The approach is further detailed in [18].

4. Filter update based upon gravity and heading updates: The differential magnetometer system provides heading updates during regular measurement stops. In the absence of forward motion, accelerometer readings only contain gravity, sensor errors and noise, but no kinematic acceleration. Collectively, the magnetic heading and stationary gyroscopic readings are used for filter updates. Furthermore, the AHRS algorithm is aided by an error approximation conducted during SF.

\subsubsection{Differential Magnetometer System}

The magnetometer system yields independent attitude data based on measurements of the surrounding magnetic field by means of scientific fluxgate magnetometers. The sensors have a measuring range of $65.000 \mathrm{nT}$, a resolution 
of $7,75 \mathrm{pT}$, and a maximum sampling rate of $100 \mathrm{~Hz}$. Sensor noise is low with 20 pT@1 Hz. These specifications assure a high resolution and a measuring range larger than the Earth's magnetic field. To provide magnetic heading, two magnetometers are used: One is integrated into the DR box aboard the IceMole, the other one serves as a static magnetic heading reference and is installed at the glacier surface (figure 1). The use of a reference measurement (instead of a reference model of the Earth's magnetic field) is advantageous, when applying the system in regions close to the magnetic pole, or when significant variations in the magnetic field are expected. For test sites relevant to the EnEx project, alterations of the magnetic field are significant. Figure 5 shows the variation of the magnetic field intensity at a glacier test site over a period of about $8 \mathrm{~h}$. It varies in the range of $40 \mathrm{nT}$, which is about $0.1 \%$ of the total magnetic field. This translates into a maximal heading error of about $1^{\circ}$.

Requirements for the application of the reference magnetometer in the field are a static position at constant attitude and a location in a magnetically relatively clean environment with few disturbances. It has to be leveled, such that its z-value (long axis) corresponds to the z-axis of the navigation frame (plumb line). Especially when being exerted to intense solar radiation, such as in the Antarctic summer, it can happen that a slow tilt is induced due to melting underneath the magnetometer's legs. This tilt translates into a small deflection of the magnetometer's z-axis with respect to the z-axis of the navigation frame. Such a deflection can be eliminated during processing of the recorded data based on an independently available measurement of the direction of gravitational acceleration (IMU). Hence, this is not critical. 


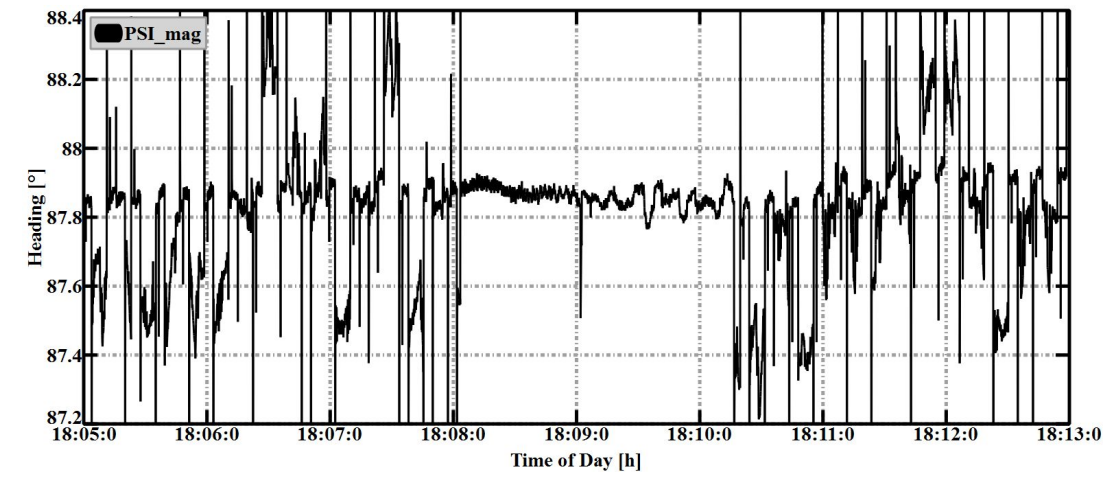

Figure 6: Magnetic heading during motion and measurement stop. Between 18:08 to 18:09 a single, and last rotation of the ice screw is visible after the side heaters were turned off. Single magnetic bursts are cut being too high $\left(>5^{\circ}\right)$ to be taken into consideration.

Instead, rotation around the z-axis would be more critical, however, this would require a z-axis torque acting on the magnetometer, which cannot easily be induced by melting. Also, short-term disturbances at the reference are very small and can be neglected. The angle between the z-value and horizontally measured values is referred to as the local inclination, the angle between sensor $\mathrm{y}$ and $\mathrm{x}$-axis as magnetic heading of the reference.

Magnetic measurements have to be corrected for distortion errors due to secondary magnetic fields induced by magnetic materials, electromagnetic sources and magnetic reluctance. This is especially true inside the IceMole. To correct for magnetic disturbances in the acquired data, a magnetic baseline model of the IceMole has been developed. Generally, the disturbance of a 
magnetic field is modeled as an ellipsoid, characterized by its eigenvectors and center. Without any disturbance, the ellipsoid is given by a centered sphere. The matrix which transforms from a centered sphere to the ellipsoid is referred to as the correction matrix and corrects for errors associated with this particular disturbance. The ellipsoid itself is determined by measurements in a special calibration unit, which is magnetically calibrated beforehand and assures a clean background. For the IceMole both static, and dynamic disturbances (and corresponding correction matrices) have been determined. Static calibration was conducted by acquisition of approximately 150 measurements, while performing various rotations through specific orientations. The ellipsoid is then given by a best-fit. Corrections have accounted for as much as $10 \%$ of the measurement value per sensor axis. To account for the dynamic disturbances, each disturber has been considered individually by measuring its isolated effect on the magnetic field. This yielded a set of correction matrices each of which is associated to one disturber. A particular IceMole configuration corresponds to a set of disturbances to be active. In order to correct for their combined error all correction matrices of the associated set are applied to the raw magnetometer data.

A significant noise level is present in the magnetic field of the IceMole, which makes data interpretation difficult. Figure 6 shows typical magnetic heading noise levels during a field test. In this common drive-cycle the measured heading noise drops significantly during a measurement stop, when at first heaters and then the motor are turned off. The increase of noise displayed in figure 7 was a result of turning on the side wall heaters. Although the induced noise is non-zero and adds a constant aberration to the heading, 


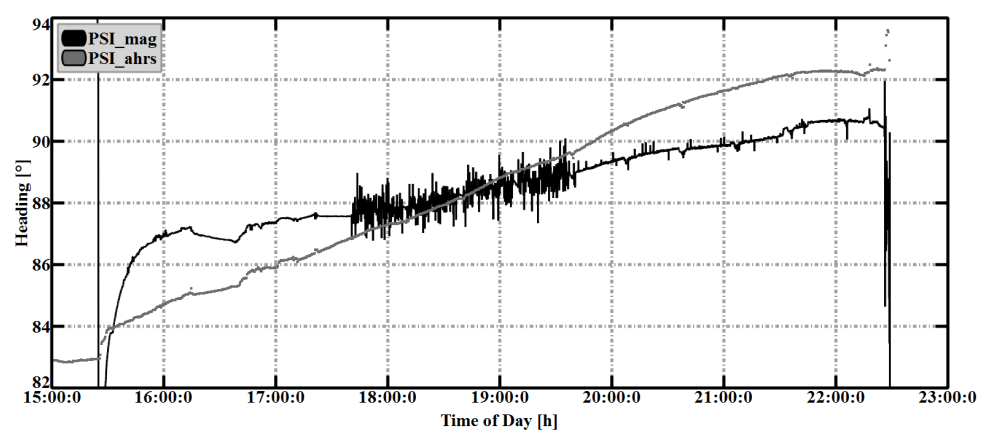

Figure 7: Comparison of uncorrelated heading data from AHRS and magnetometer. While AHRS heading drifts over time, the magnetic heading has non-observable modeling errors. The period of increased noise level between 17:45 and 19:30 indicates the use of the side wall heaters.

it can be eliminated by using a low-pass filter and an estimate filter to reduce the error. In addition to that, no significant remanence appears from the induced magnetic fields. Though an induced magnetic field can be observed from the stepper motor and heaters, this generates no significant remanent magnetisation onto the materials used in the IceMole. The induced magnetic field of the stepper motor or heaters disappears after short time. Based on the detected noise the covariances for later fusion are adjusted.

The magnetometer raw data recorded on-board the IceMole yields a magnetic field vector that indicates the attitude of the fluxgate sensor relative to the local magnetic field. To determine the magnetic heading from the measured magnetic field vector, it is rotated into a North-East-Down (NED) coordinate system. The transformation matrix (direction-cosine matrix) relies on an independent measurement of the gravity vector by the IMU. In a next step, the rotated vector is projected onto the xy-plane of the NED coordinate system and evaluated as the magnetic heading $\Psi$. With the 
local declination and inclination (DI), the magnetic heading $\Psi$ can finally be converted into geographic north. Prior knowledge of DI can be acquired by external alignment given by AHRS (standard procedure during EnEx operations) or SF, or alternatively, by using less precise look-up tables. Any reoccurring alignments during a mission improves the initially determined DI and will have a positive effect on the accuracy of the navigation results. It can also be used to model the surrounding environment of the IceMole during mission and update the IceMole magnetic model for compensation. However, reliability of DI data is depending on both external alignment as well as magnetic sensor variances and should therefore only be acquired once the local magnetic field is no longer influenced by disturbances, e.g. induced by the IceMole launch pad.

\subsubsection{Collective AHRS and Magnetic Data Processing}

Both the attitudes determined from AHRS, and the magnetic heading have complementary advantages and drawbacks. The error of an AHRS-onlyheading varies depending on its alignment state. Starting from a small error with a new alignment, the error increases during a mission and suddenly drops during the realignment. Though in principle, magnetometer-only-headings would have low noise and negligible ascending errors, there is a significant distortion of results due to the presence of a-priori unknown magnetic sources and secondary magnetic fields during run-time. These previously unknown errors depend on position, attitude and state of the Icemole. The impact of the heating system on the noise level of the magnetometer sensors is visible in figure 7. This figure also shows the differences between AHRS and magnetometer heading. State driven changes of the magnetometer reading 

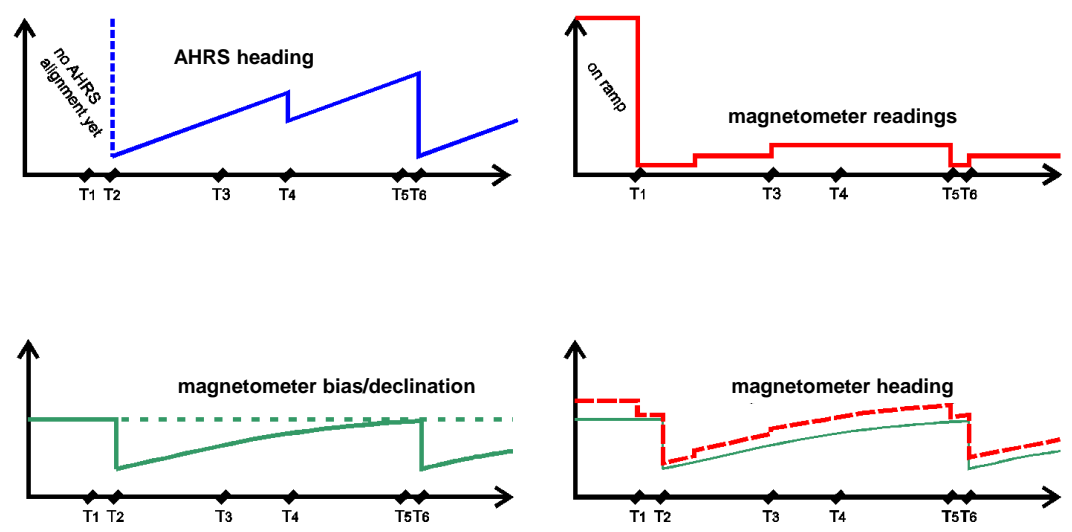

Figure 8: Qualitative comparison of heading variances: The upper left plot indicates the increasing covariance errors of the AHRS heading during mission due to drift between realignments of the system (initial alignment in T1, realignment in T4 and T6). The upper right plot indicates the variance of the magnetometer heading due to system-noise or external disturbers, e.g. the starting ramp between $\mathrm{T} 0$ and $\mathrm{T} 1$ or low noise at measurementstops (T5-T6). The lower left plot shows the variance of the magnetometer bias (DI) estimation, which drops to the level of realignment data at T2 and T6 (but not in T4 with non improving correction data) and increases in between corrections due to physical movement into other areas of unknown DI (limited by look-up table values). Finally, the calculated and passed heading variance equals a superposition of the bottom left and top right variances as shown in the bottom-right plot.

on a conceptual level are shown in figure 8, upper-right. The increase of uncertainty due to passing an unknown disturber is shown in figure 8, lowerleft. To provide one attitude result for further processing in the DR algorithm, AHRS attitude, magnetometer heading and reference corrections are fused based on variances of the measurements and states of the relevant subsystems. The full conceptual schematic indicating the qualitative characteristics of the AHRS and magnetometry fusion (AHRS/MAG) is given in figure 8. 


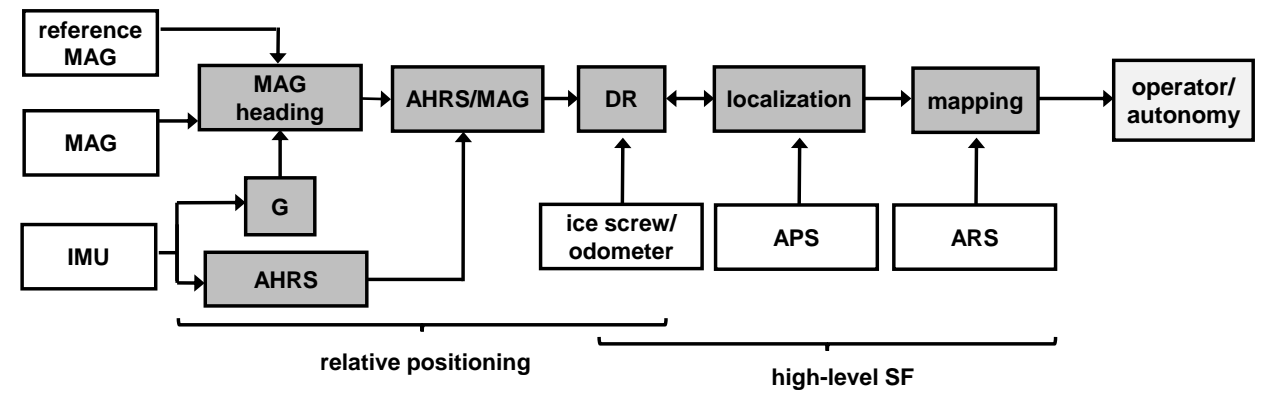

Figure 9: Overview of the navigation data process chain: The white boxes indicate sensor input data, the light grey box indicates output and the dark grey boxes indicate data processing steps. The IMU raw data is processed within the AHRS. It furthermore determines the gravity vector $(\mathrm{G})$, which is used to calculate magnetic heading (MAG heading) from magnetometer data (MAG, reference MAG). Both results are combined yielding the drift stabilized attitude of the IceMole (AHRS/MAG). Next, DR based on the attitude estimate AHRS/MAG and information on the traveled distance yields a predicted state of the IceMole. Subsequently, the predicted state is corrected with APS measurements. The corrected state is both used in the next prediction step, and enables computation of a global 3D map from ARS measurements. State of the IceMole as well as the computed map are visualized for the operator and can be used for trajectory planing and autonomous navigation.

\subsubsection{Dead Reckoning Algorithm}

The main steps of the DR algorithm and its connection to the global Sensor Fusion (SF) are displayed in figure 9. DR is calculated from AHRS/MAG fusion and the axial speed data generated of the ice screw turn rate. A launchpad odometer yields information on the used rope length and provides additional input. DR is conceptually a part of the relative position and attitude determination (section 3.1). Due to its role as predictor in the calculation of the final IceMole state, however, its effective implementation is integrated into the high-level SF (section 3.4), in which results of the DR are 
also further processed. Reliable external updates via the SF from e.g. APS are available only at certain points in time, hence the AHRS/MAG fusion represents the major source for the navigation system in between external updates. Both AHRS/MAG, and DR have been tested successfully under laboratory conditions and in various field campaigns. The system was able to perform initial alignment as well as continuous tracking of the probe's attitude and position. Updates during measurement stops were used successfully for the stabilization of the heading drift. Particular scenario examples are further outlined in the section on field test results.

\subsection{Acoustic Positioning System}

\subsubsection{Principle of the APS}

The Acoustic Positioning System (APS) provides data on the absolute position of the IceMole within a volume of glacier ice [23]. Subsets of this data are used by the SF to correct the drift of the position provided by DR (section 3.1). As stand alone system the APS is based on trilateration which is the determination of the unknown position of an object, here the probe within ice, by measuring the distances to at least three other objects of known position [24]. These distances are obtained by measuring the propagation times of pulsed ultrasonic signals between the emitters at the ice surface and the receivers within the probe [23] (figure 10). Given the signal propagation times, the speed of sound in ice and the known emitter positions, the position of the probe can be reconstructed for a specific time. Monitoring the position over time yields the trajectory of the probe. 


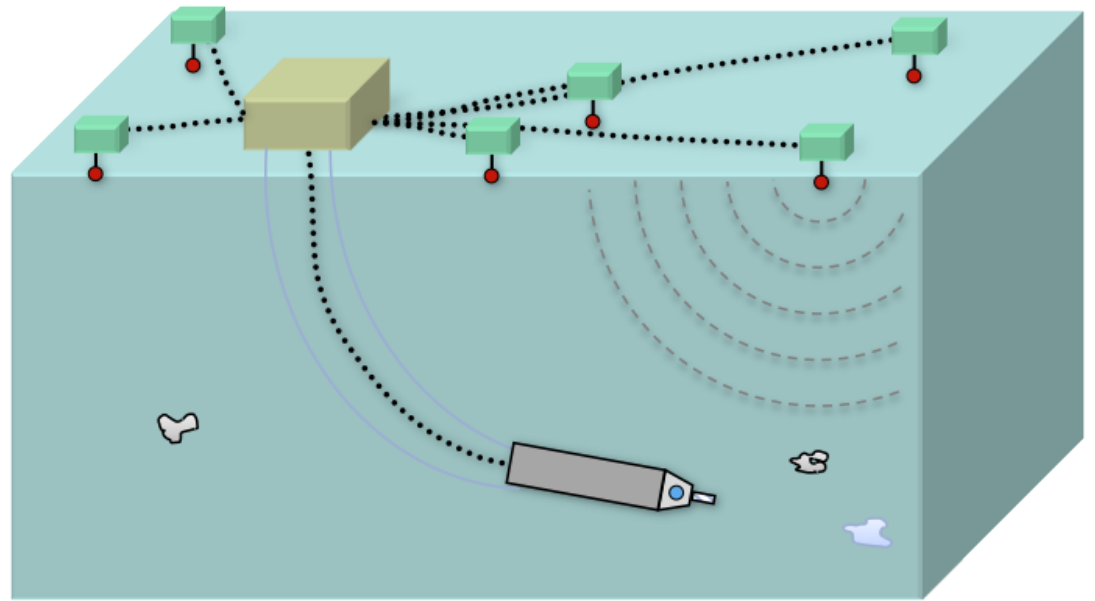

Figure 10: Principle of the APS: The absolute position of the probe within ice is determined by trilateration. For this purpose the distances between the emitters at the surface and receivers within the probe are determined by measuring the propagation times of pulsed ultrasonic signals.

\subsubsection{Acoustic Properties}

In order to achieve accurate acoustic in-ice navigation it is essential to know the physical parameters regarding the speed of sound as well as the attenuation in ice. This project was focused on the properties of terrestrial ice. Future missions to Enceladus have to be based on sufficiently autonomous instruments capable of adopting algorithms to the specific ice structure and quality and calibrate them with parameters determined at the landing site. Generally, sound waves propagate in solid materials as pressure and shear waves [25]. Pressure waves travel much faster in ice than shear waves and are therefore the first to be detected [26]. For pressure waves the speed of sound in glacier ice is about $3600 \mathrm{~m} / \mathrm{s}^{2}$. However, due to inhomogeneities

\footnotetext{
${ }^{2}$ Measured during the field campaigns carried out within the EnEx project.
} 
and density gradients in glacier ice the speed of sound is not constant but depth dependent $[27,28,29]$. Strength and therefore quality of the received acoustic signals is influenced by attenuation which is composed of absorption and scattering. While the first reduces the received amplitudes and limits the sensitivity, scattering leads to changes in arrival times. Like the speed of sound, attenuation varies with depth and depends on ice structure and inhomogeneities. Compact, clear ice as it is found deep below the glacier surface attenuates less than firn ice, which contains many gas inclusions [28]. With the South Pole Acoustic Test Setup (SPATS) it was found that at a depth between $150 \mathrm{~m}$ and $500 \mathrm{~m}$ the attenuation length is about $300 \mathrm{~m}$ for frequencies between $10-30 \mathrm{kHz}$ [30]. However, attenuation in ice is in general expected to depend on the frequency of the acoustic signal [31]. Low ultrasonic frequencies show less attenuation compared to higher ultrasonic frequencies, whereas higher frequencies provide better spatial resolution.

\subsubsection{System Design}

The APS consists of six ultrasonic emitters, four receivers, a signal generation system and a data acquisition (DAQ) system [23] (figure 11 for a system overview). The signal generation system is situated at the glacier surface and the ultrasonic emitters are inserted into the glacier at a depth of about one meter. The receivers and the DAQ system are integrated into the IceMole head (figure 3) and the IceMole payload bay (figure 1), respectively. The APS is optimized for signal frequencies of about $18 \mathrm{kHz}$.

The acoustic emitters used in the APS are spherical, omnidirectional, piezoelectric (lead zirconate titanate) sonar pulse transducers. The net of six emitters is connected and controlled via the Field Programmable Gate 
Array (FPGA) based Central Transducer Unit (CTU), which generates pulsed signals. The signal from the CTU is amplified by the front end unit and then passed onto the emitter. Additionally, the emitters can be used as receivers which allows measurements from one emitter to another to investigate ice properties, as detailed in the results section 4.4. The CTU also ensures synchronization between the signal generation and the IceMole by a strobe signal distributed via a dedicated cable. The accurate position of each emitter is needed for trilateration and is determined via GNSS RTK $(\sigma=2.5 \mathrm{~cm})$.

The APS receivers consist of a piezoelectric disk (lead zirconate titanate) and specially dedicated front end electronics. Both, the piezo disk and the front end electronics are encased by a metal housing to reduce electromagnetic noise pick up. Good acoustic coupling of the piezo disk and the IceMole head is achieved by pressing the piezo disk to the melting head. The front end electronics contains two amplification stages including two analog filters, one broad and one narrow passband. Both channels are digitized by analog-todigital (ADC) converters with 12 bit resolution and a sampling rate up to $2 \mathrm{MS} / \mathrm{s}$.

Data acquisition is performed by the FPGA (Spartan-6 SLX 16) based APS DAQ system. The DAQ system (figure 12) simultaneously reads the digital outputs from the four receivers, stores the recorded waveforms and then transfers these to an embedded PC (Raspberry Pi). There are two possible digitization modes, one with a lower sampling rate but a higher number of recorded waveforms per emitter and one with a higher sampling rate but less recorded waveforms. The first mode records 64 waveforms per emitter with a sampling rate of $500 \mathrm{kS} / \mathrm{s}$, while the second mode records 32 


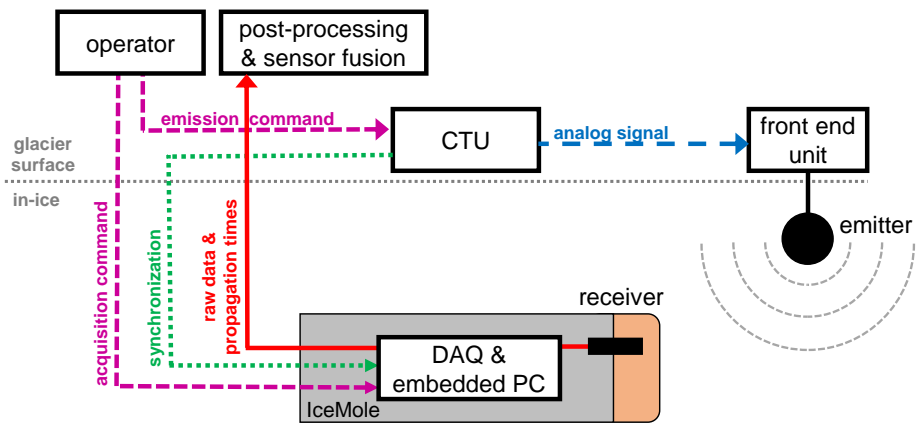

Figure 11: APS system overview: The signal generation system is situated at the glacier surface and the ultrasonic emitters are situated about one meter below the surface. The receivers are integrated into the IceMole head and the DAQ system is integrated into the IceMole payload bay.

waveforms per emitter with a sampling rate of $2000 \mathrm{kS} / \mathrm{s}$. In both cases the data acquisition window is about $32 \mathrm{~ms}$ starting one millisecond before the acoustic signal is emitted. This ensures that one millisecond of pure noise is obtained in each waveform. The DAQ system is synchronized with the CTU with an accuracy of better than $1 \mu \mathrm{s}$.

Data preprocessing is performed by an embedded PC. To reduce noise the waveforms of each receiver are averaged and filtered using a band pass filter. The signal propagation time is determined by searching for the earliest point in time, when the noise level is surpassed by a factor of five. The noise level is defined as the standard deviation of the first millisecond of data. Raw data as well as signal propagation times are transferred to a central database at the surface via TCP/IP.

The APS is controlled by the operator via the EnEx run control software (section 2.2). Once a measurement cycle is initiated, signal generation within the CTU is triggered and simultaneously data acquisition is started. 


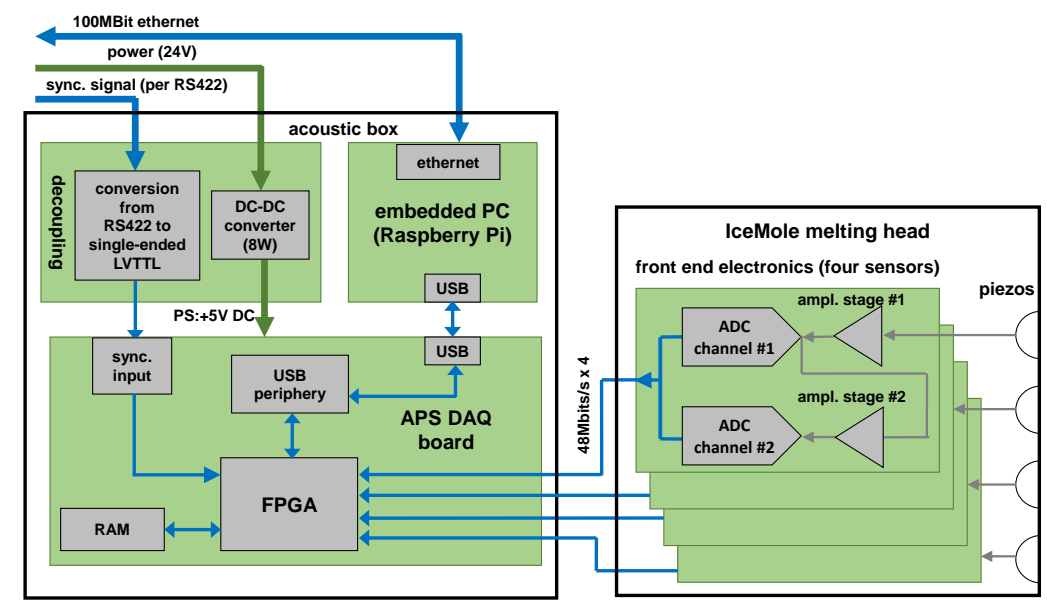

Figure 12: Overview of the APS DAQ system for acquisition, analog and digital processing of the acoustic waveforms. Piezo disks and custom front end electronics are integrated into the IceMole melting head (box on the right), online processing is conducted in the IceMole's acoustic box.

At the surface, data quality can be further improved in contrast to online processing by applying advanced de-noising techniques to the recorded data. For this purpose a combined de-noising method based on both wavelet transform and infinite impulse response filters [32, 33] has been developed. To ensure a reliable trilateration only signals are considered, which pass iterative consistency tests with respect to the reconstructed position.

The APS has been successfully tested in the laboratory, in full system swimming pool tests and in several field campaigns within both temperate and non-temperate glaciers. Further details can be found in section 4 .

\subsection{Acoustic Reconnaissance System}

The Acoustic Reconnaissance System (ARS) is designed to detect possible obstacles or regions of interest in front of the probe. This fore-field informa- 


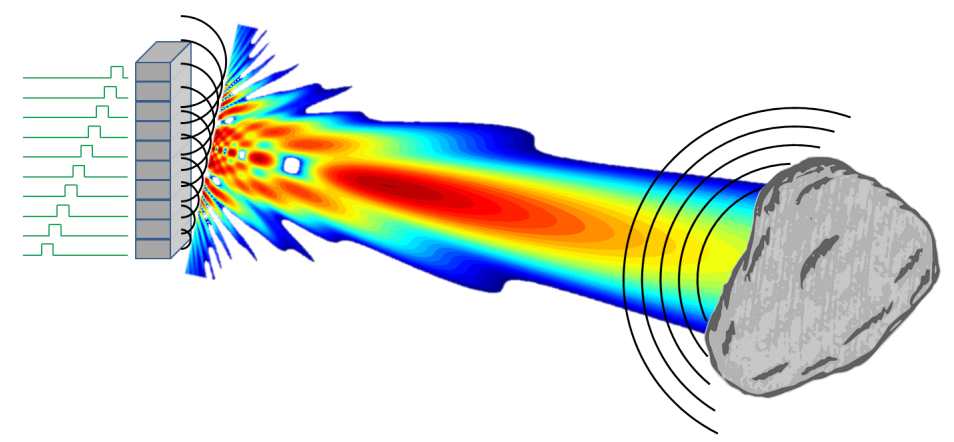

Figure 13: Principle of the ARS: Acoustic waves are emitted by piezo transducers. The electronic system is switched to receiver mode and echoes reflected at structural inhomogeneities (e.g. stones, bubbles etc.) are then acquired by the same piezos. The arrangement of several piezo transducers in an array structure allows for steering and focusing of the acoustic lobe due to phase shifted emission of acoustic waves.

tion is acquired by sonography (figure 13), primarily known due to medical applications [34, 35] or material inspection [36, 37]. ARS uses emission of phased acoustic signals and the recording of respective echoes. Any disturbances within the ice like crevasses, stones, water or air filled pockets create echoes of the emitted signals due to the difference in acoustic impedance of the different media. Imaging is based on the amplitudes and time delays of the received echoes. To enable a scanning of the environment, piezo arrays are driven with phase shifted signals. This results in the formation of an acoustic lobe. By variation of the phase shift between the single piezo elements the direction and focal distance of the lobe can be adjusted [38, 39].

As in case of the APS, the performance of the ARS highly depends on the physical properties of the ice. The general dependencies are described in section 3.2.2. Since the ARS uses higher signal frequencies than the APS the increased acoustic attenuation has an even higher impact on signal 
propagation. Due to the direct contact with ice, the physical properties of ice and the harsh environment in which the phased arrays have to work, e.g. high temperature gradient and high pressure on the active surface, a design had to be developed with specifications differing very much from typical off the shelf products as used in medical and material testing applications.

\subsubsection{System Design}

In the melting head four linear phased arrays (Manufacturer: Imasonic, Voray-sur-l'Ognon, France) are integrated crosswise around the ice screw leaning $7.2 \mathrm{deg}$ to the outside (figure 2 and 3 ). The phased arrays developed for this application consist of 16 elements with a resonance frequency of $780 \mathrm{kHz}$ inside a housing made of the same material as the melting head. The active area is made of epoxy resin to adjust the acoustic impedance to ice impedance. This configuration enables a steering of the acoustic lobe of more than \pm 45 deg with an overlap of the field of view directly in front of the probe.

Pulse pattern generation and data acquisition is performed by one FPGA based electronics per array to achieve phase shifting at very high precision. An output stage transfers these logic pulses into high voltage and addresses each of the 16 piezo elements of each array separately. Immediately after all pulses are sent, the acquisition of acoustic signals arriving at 8 of the 16 array elements starts. Sampling is performed by amplification and high speed digitization of 16 kilosamples at $2 \mathrm{MS} / \mathrm{s}$, resulting in a maximum acquired distance of approximately $15 \mathrm{~m}$ in water ice. An embedded PC is used to handle the data and communicate with the surface.

The ARS system is controlled via the EnEx run control software. A 

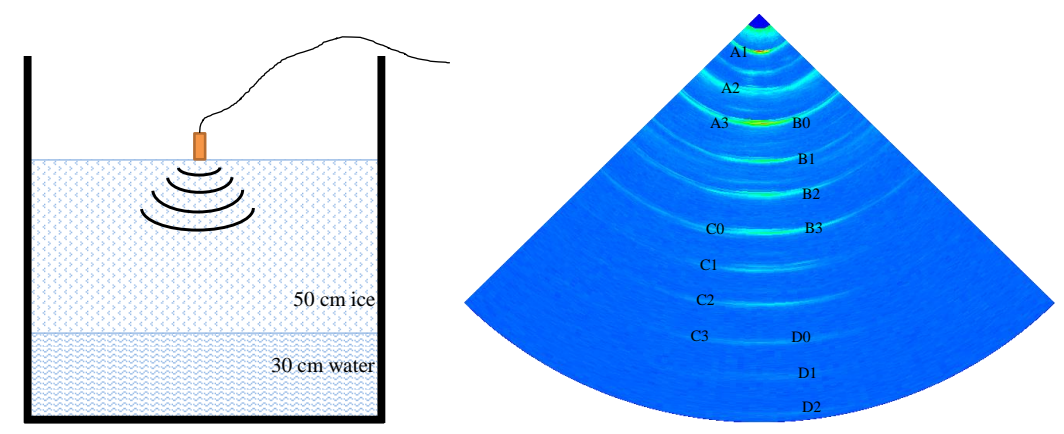

Figure 14: Left: Laboratory setup with a volume of $3 \mathrm{~m}^{3}$. This contains a $50 \mathrm{~cm}$ layer of bubble free ice above a $30 \mathrm{~cm}$ layer of liquid water. On top of the ice layer a phased ultrasonic array is positioned pointing into the ice. Right: A standard ARS B-scan. The labels A to D represent multiple passages of the wave between the ice surface and the bottom of the container, where index 1 indicates the first reflection from the ice/water interface. Index 2 and 3 are multiple reflections between top and bottom of the ice layer and index 0 indicates the reflection from the bottom of the container superposed with index 3 .

typical ARS measurement stop performs a scan from -45 deg to +45 deg in 1 deg steps for each array. The data is transferred to a central database at the surface via TCP/IP (section 2.2). The acquired data is phase corrected and averaged for visualization. Absolute values of the signals are low pass filtered with a cutoff between $100 \mathrm{kHz}$ to $500 \mathrm{kHz}$ by applying a 3rd order Butterworth filter to create the envelope and then plotted in a 2D intensity graph. Finally, the graph is transformed to a polar mapping representing the scanned sector (B-scan).

\subsubsection{Laboratory Tests}

The system was tested in the Aachen Acoustic Laboratory at RWTH Aachen within a Polyurethane container with bubble-free ice volumes up to 
$3 \mathrm{~m}^{3}$ (figure 14). Due to the limited volume numerous artifacts are measured, as the acoustic wave travels multiple times back and forth. During the test (figure 14) the volume was not entirely frozen and thus several echoes are visible during each passage of the sound wave between different layers of media. The main echoes visible are the reflection from the ice/water interface and from the bottom of the container. As the speed of sound in bubble-free ice $\left(3838 \mathrm{~m} / \mathrm{s}\right.$ at $\left.0^{\circ} \mathrm{C}[26]\right)$ is more than twice the speed of sound in water $\left(1400 \mathrm{~m} / \mathrm{s}\right.$ at $\left.0^{\circ} \mathrm{C}[40]\right)$, the first reflected signal travels multiple times between the top of the ice layer and the bottom of the ice layer before the echo from the bottom of the container is detected. This pattern can be observed at least four times, meaning that the signal traveled in total through $4 \mathrm{~m}$ of ice and $2 \mathrm{~m}$ of water. Moreover, the signal was attenuated at the ice/water interface ( $40 \%$ loss), the reflection at the bottom of the container ( $80 \%$ loss) and the phase transition from water to ice ( $40 \%$ loss) during each passage through the container.

During a second test (figure 15) one phased array was integrated into the IceMole in horizontal position and submerged in a swimming pool. Due to the large volume of the pool, fewer artifacts were induced by wall reflections. Spherical objects with a diameter of $20 \mathrm{~cm}$ filled with air or concrete were spread throughout the pool as phantoms. Due to the lower speed of sound compared to ice, the maximal acquirable distance is limited by the acquisition window to about $6 \mathrm{~m}$. All objects can be detected as reflections in the ARS B-scan (figure 15). The difference in acoustic impedance of water as compared to air produces a single, very well defined echo peak (total reflection) of the air filled phantoms whereas the concrete filled phantoms produce two peaks 
${ }_{618}$ (front and back side) of lower amplitude. 

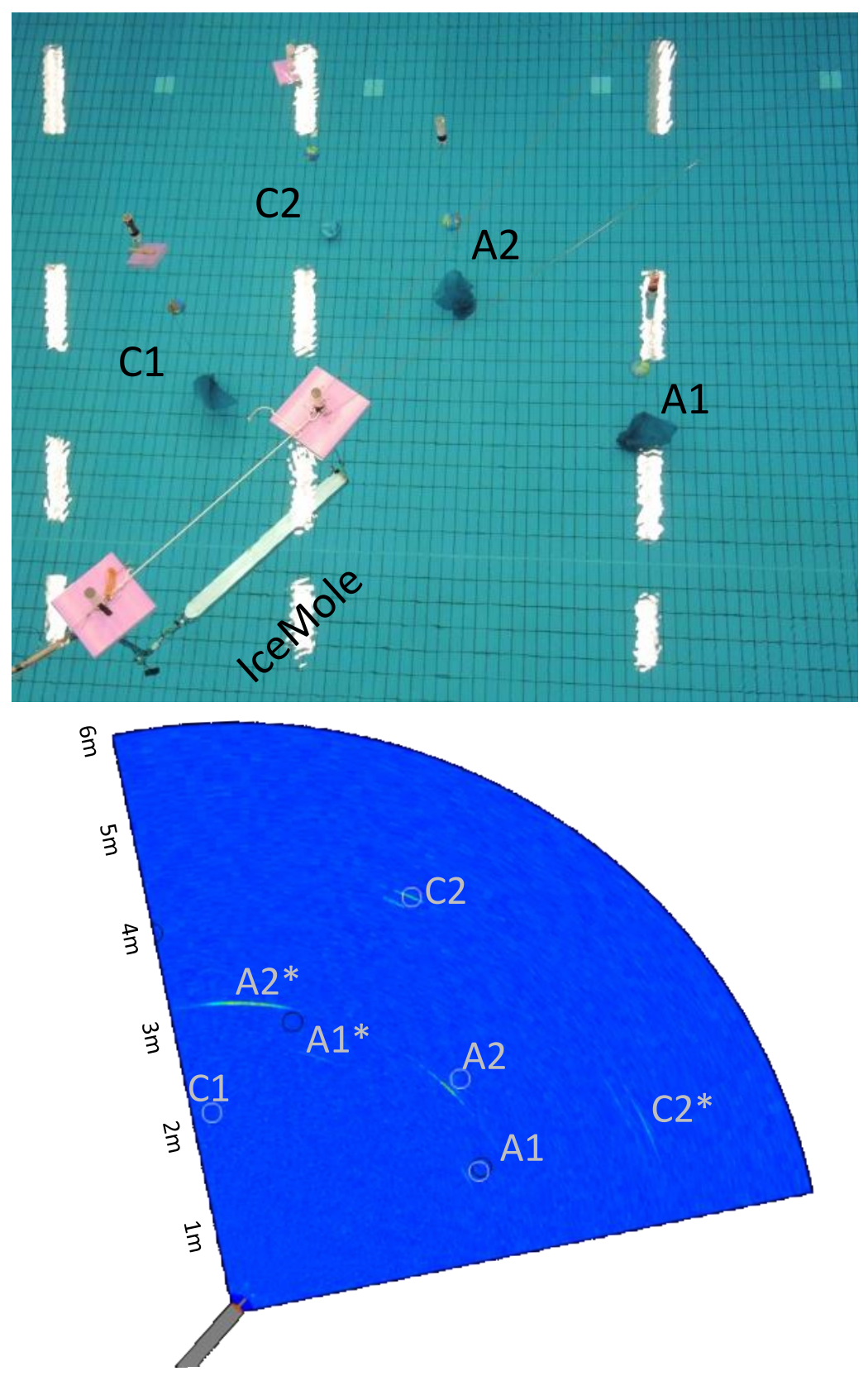

Figure 15: Top: Test setup in a swimming-pool $\left(17.7 \times 14.34 \mathrm{~m}^{2}\right)$ with spherical air $(\mathrm{A} 1$, A2) and concrete $(\mathrm{C} 1, \mathrm{C} 2)$ obstacles with a diameter of $20 \mathrm{~cm}$. The IceMole contained one phased array with horizontal scanning direction. Bottom: Scanned data $( \pm 45 \mathrm{deg}, 6 \mathrm{~m}$ sampling window); white circles mark obstacle positions measured at the water surface $( \pm 5 \mathrm{~cm})$. Side-lobe echoes (marked by $\left.{ }^{*}\right)$ are 3 letected at the same distance and at a fixed angle deviation from the true object. Additionally the side lobe echoes are blurred and much lower in amplitude. Therefore, these side lobe echoes can be identified as ghosts which can be filtered out by advanced signal processing tools. 


\subsection{Multi-sensor Fusion and High-level Postprocessing}

Multi-sensor fusion and high-level postprocessing enable the use of IMU, MAG, DR, APS and ARS data for IceMole navigation purposes. In particular, this provides an estimate of the current state $x_{t}$ (position and attitude) of the probe and a global map $Y$ of the environment. An overview of the sensor fusion and the data processing chain is shown in figure 9 .

\subsubsection{Data Handling and Time Reference}

The data produced by all sensors and subsystems of the navigation suite are stored in and distributed by a central database running on the navigation notebook at the glacier surface. It provides a standardized interface via TCP/IP and allows other subsystems to access not only the current but also past data. This is especially important when systems run with different data acquisition frequencies. In addition, the integrated mechanisms for dumping and restoring the whole database or selected tables are used for backups and post-processing. In practical tests this solution turned out to be fast enough to handle both, small data with a high frequency (e.g. IMU data with $200 \mathrm{~Hz}$ ) as well as large data with a low frequency (e.g. APS and ARS raw data), simultaneous and in real-time. Furthermore it is important that all subsystems share the same time reference. A GPS receiver is used to retrieve the current time. Subsystems are synchronized via NTP (network time protocol). All data packets are time-stamped.

\subsubsection{Position and Attitude Estimation}

Because all errors are assumed to be Gaussian, an extended Kalman filter (EKF) is used to estimate the state. For the prediction step, the traveled dis- 
tance between time $t$ - 1 and $t$ is computed from the control commands $u_{t}$ given to the screw step motor. Since gear transmission ratio and the pitch of the ice screw are known quantities, distance traveled can be calcuated from standard motion equations. For this calculation the screw is assumed to be nearly slip-free with additive Gaussian noise. In the correction step, the predicted state $\bar{x}_{t}$ is corrected using the preprocessed measurements $z_{t ; q}$ resulting from the AHRS/MAG fusion (section 3.1.1 and 3.1.2) and the measurements $z_{t ; p}$ of the APS (section 3.2). Because $z_{t ; q}$ provides an attitude quaternion together with a covariance matrix describing the underlying uncertainty, it can be incorporated using the usual Kalman equations. The propagation times $z_{t ; p}$ measured by the APS have to be converted into distances by multiplying them by the speed of sound in ice. In a next step a position estimate based on trilateration techniques (e.g. Bancroft's method [41] or least-squares) is used for correction. However, the geometric constellation of the acoustic emitters is not optimal for trilateration, because they are arranged approximately in a single plane which results in large positioning errors from small measurement errors. In addition, trilateration in 3D space needs at least three distance measurements (if one solution can be excluded as implausible), but it can happen that only the signals of one or two emitters are received, even when all emitters are active. To overcome these limitations, a tightly coupled update [42] is used instead of trilateration. Thereby, the non-linear distance errors for all received emitter signals are calculated from the measured and expected distances. Expected distances are given by the Euclidean distance between the estimated state and the emitters' positions. By means of a first order Taylor series expansion, the estimated errors are used to correct the relevant 


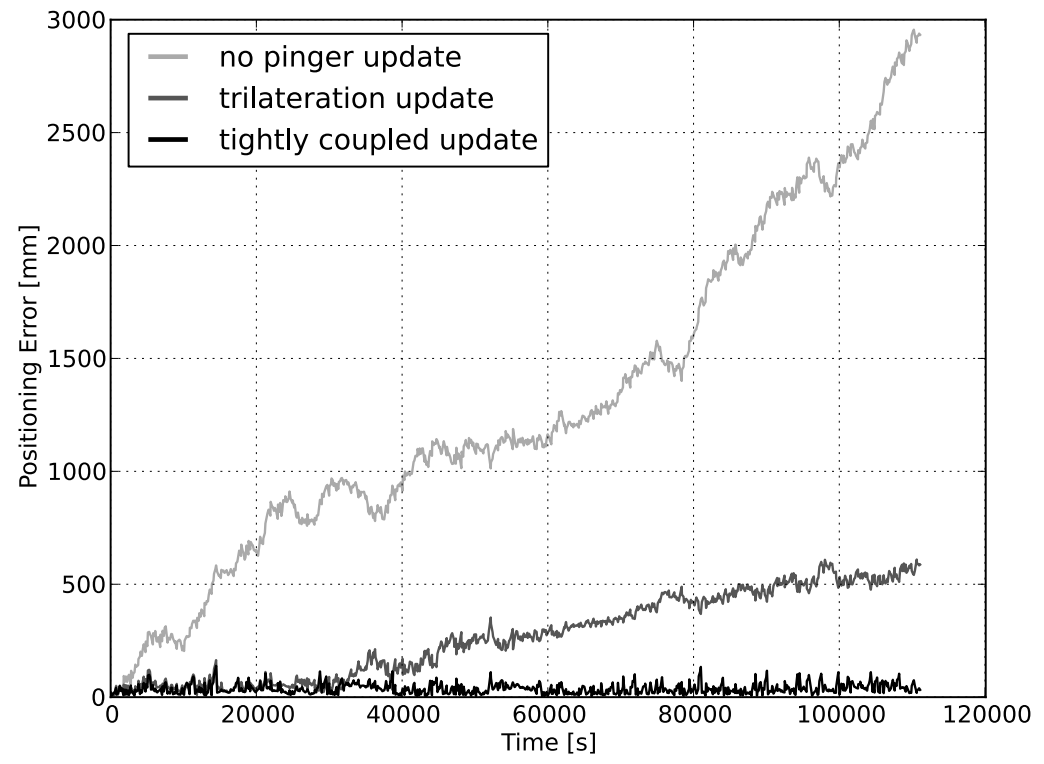

Figure 16: Positioning error for different methods to correct the state estimate with the APS based on simulation data [18]. The different lines show the error without integrating the measurements of the APS (no pinger update), the result for the update with trilateration, and the the error of the tightly coupled update.

components of the state vector. This strategy reduces the positioning error significantly, as shown in figure16. It allows the fusion algorithm to perform state correction even with a single APS measurement.

\subsubsection{Mapping of the Environment}

The environment map is computed from the measurements of the ARS (section 3.3). The field of view of its four sensors covers two $2 \mathrm{D}$ cutting planes $(\mathrm{x} / \mathrm{y}$ and $\mathrm{x} / \mathrm{z})$ in front of the IceMole. These local maps are fused over time into a global 3D map. A common approach to solve this task is grid mapping, which was successfully applied to a wide range of robotic applications where the map $Y$ is discretized into equal-sized cells $Y_{i}$ which 
can be either occupied or empty (denote by $o$ and $e$ ). In a probabilistic grid map, a single probability $P(o)$ is used to model the state of a cell. The approach used within this project however, relies on an evidential grid map [43, 44, 45]. It is based on the belief function theory [46] (also called Dempster-Shafer theory), a generalization of the classical probability calculus, and uses belief functions to represent the state of each cell. This allows for probability mass assignments not only to the singletons $o$ and $e$ but also to the union $\Theta=\{o, e\}$ and the empty set $\emptyset$. This has the practical advantage that it makes different dimensions of uncertainty explicit [47]. A lack of measurements for example, can be expressed by assigning mass to the union $\Theta$ and conflicting evidence can be expressed by assigning mass to the empty set $\emptyset$. In the probability framework, both cases would lead to an uniform distribution $P(o)=P(e)=0.5$ and are therefore indistinguishable. Figure 17 shows an evidential grid map and its probabilistic counterpart. Autonomous navigation as well as a human operator can take advantage of this additional information during the exploration of an unknown environment. 


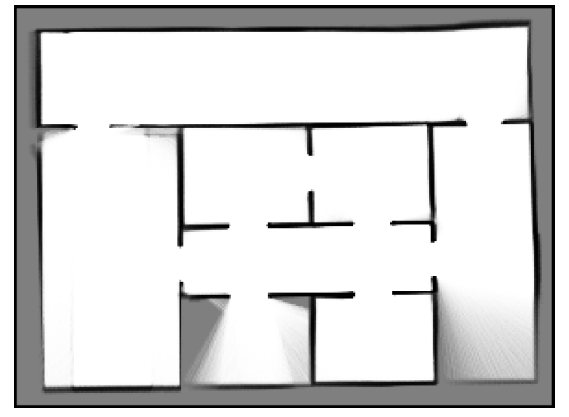

(a) Probabilistic grid map: $P(o)$

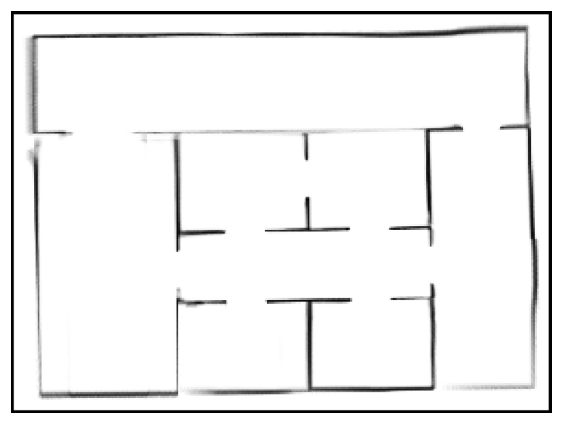

(b) Evidential grid map: $m(o)$

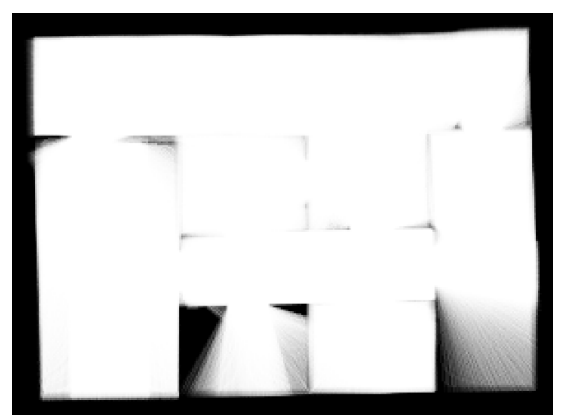

(d) Evidential grid map: $m\left(\Theta_{Y}\right)$

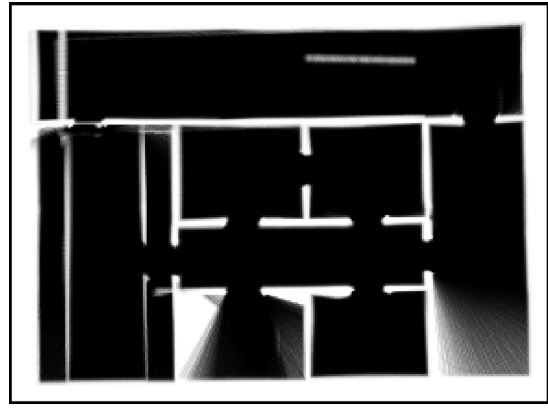

(c) Evidential grid map: $m(e)$

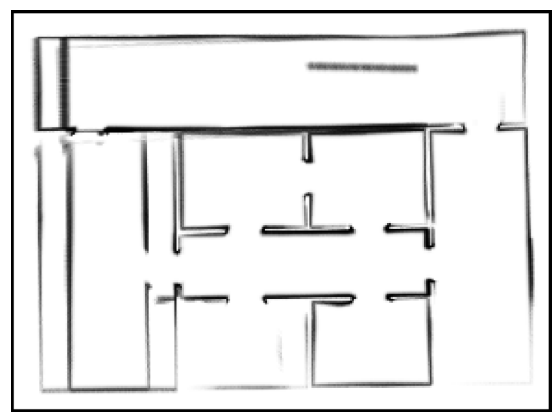

(e) Evidential grid map: $m(\emptyset)$

Figure 17: Comparison between a probabilistic and an evidential grid map computed from the sensors of a simulated robot [47]. Plot (a) shows the probabilistic grid map, where black indicates occupied areas (high probability for $o$ ), white empty areas (high probability for $e$ ), and gray uncertain areas (probability uniformly distributed between $o$ and $e$ ). Plots (b) to (e) shows different mass components of the evidential grid map, whereat (b) shows occupied areas (mass on $o$ ), (c) empty areas (mass on $e$ ), (d) unobserved areas (ignorance, mass on $\Theta$ ), and (e) areas with conflicting measurements (mass on $\emptyset$ ). Black represents a mass value of one and white represents zero. $42_{\mathrm{n}}$ the evidential grid map it is thus possible to distinguish between different dimensions of uncertainty and it provides significantly more information. 


\subsubsection{Simultaneous Localization and Mapping (SLAM)}

Although the task of exploring an unknown environment can be viewed as two separate problems (localization and mapping), they are not independent of each other. Rather it is a joint estimation problem commonly known as simultaneous localization and mapping (SLAM) [49]. A successful approach to solve the SLAM problem is the FastSLAM algorithm [50] that uses a RaoBlackwellized particle filter to estimate the joint distribution. We proposed a generalization for evidential grid maps in [51].

In first experiments, the evidential FastSLAM algorithm was successfully applied to the task of navigating a melting probe through deep ice [48] and the results are shown in figure 18. The estimated trajectories for different sensor combinations are shown in figure 19. 


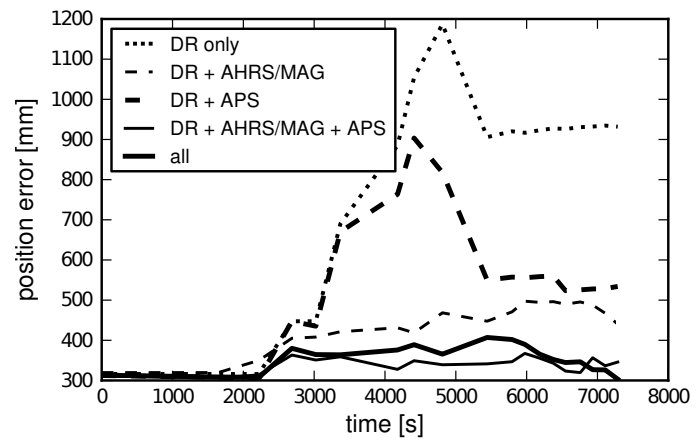

(a) Positioning error

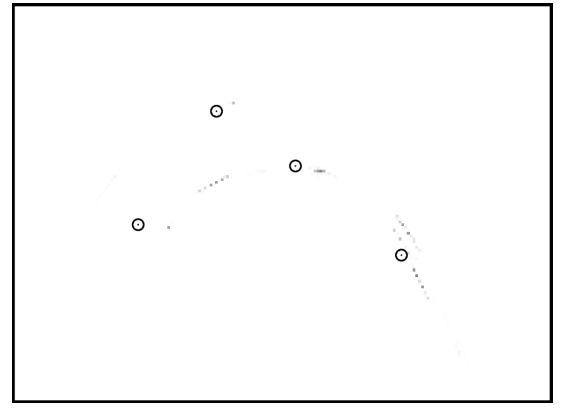

(b) Evidential grid map: $m(o)$

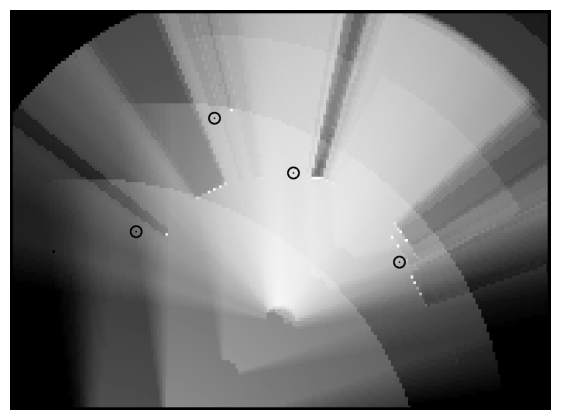

(d) Evidential grid map: $m\left(\Theta_{Y}\right)$

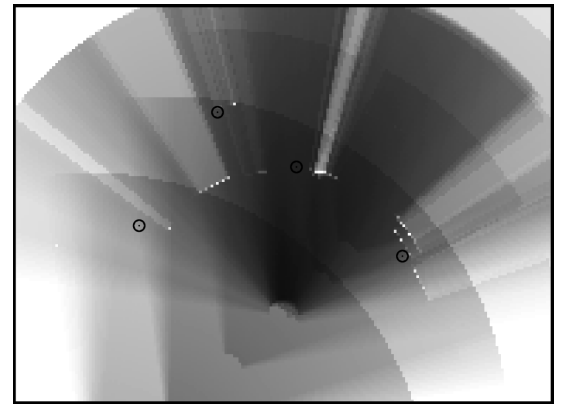

(c) Evidential grid map: $m(e)$

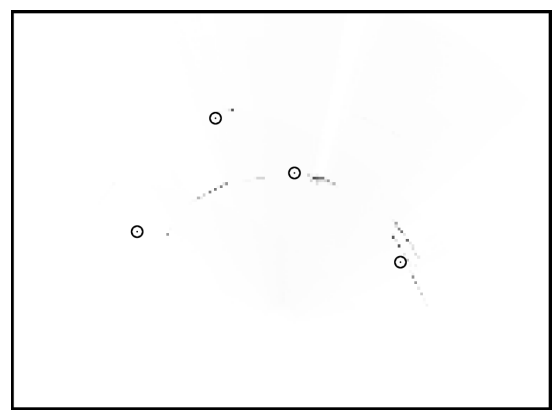

(e) Evidential grid map: $m(\emptyset)$

Figure 18: Results of the evidential FastSLAM algorithm during a pool test with the melting probe [48]. Plot (a) shows the localization error for different sensor combinations, where DR stands for dead reckoning using the screw feed only with fixed heading, AHRS/MAG for the fusion of AHRS and magnetometer data, APS for results from the acoustic trilateration, and all for the combination of all sensors including the correction with the map computed from ARS measurements. Plot (b) to (e) show the different mass components of the evidential grid map (cf. figure 17) computed by the algorithm. The four circles in the plots show the ground truth positions of the obstacles (cf. figure 15). 


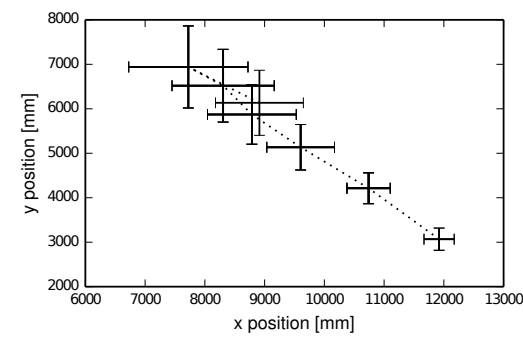

(a) DR only

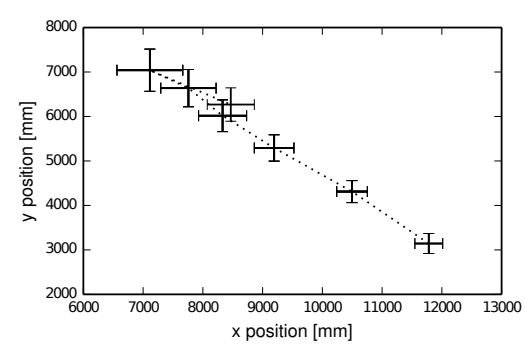

(c) DR + APS

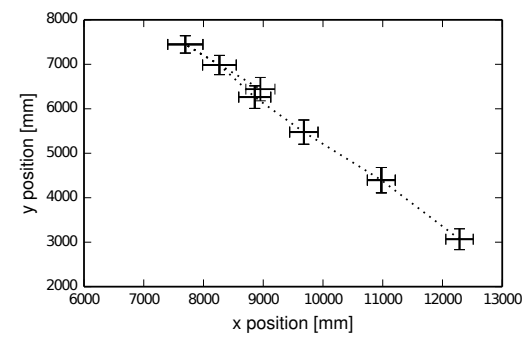

(b) DR + AHRS/MAG

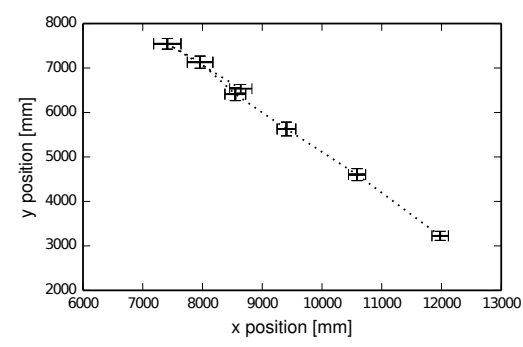

(d) all sensors

Figure 19: Estimated trajectories during a pool test for different sensor combinations. The dotted line in each plot corresponds to the estimated path and the error bars show the position uncertainty (standard deviation) estimated by the particle filter. (a) shows the result for DR using the screw feed only, (b) shows the result for DR with AHRS/MAG attitude corrections, (c) shows the result for DR with APS position corrections, and (d) shows the result with all available sensors, namely screw feed, AHRS/MAG, APS as well as ARS corrections wrt. the estimated map. DR-only has the highest estimated uncertainty, followed by DR + APS, DR + AHRS/MAG, and the combination of all sensors with the lowest estimated uncertainty. The latter corresponds to the real localization error (cf. figure $18(a))$. 


\section{Field tests}

The EnEx system has been tested in four field campaigns of increasing system complexity reflecting the different development phases of the project. Two field tests took place on the Morteratsch Glacier in the European Alps and two field tests in the McMurdo Dry Valleys, Antarctica. Relevant differences between both test regions are:

- Glacier ice temperature: Ice temperature of Morteratsch Glacier is homogeneously close to the melting point of $0^{\circ} \mathrm{C}$, whereas ice is much colder at Taylor and Canada Glacier in Antarctica (as low as $-17^{\circ} \mathrm{C}$ ).

- Proximity to the pole: The latitude of Morteratsch Glacier is about $46,4^{\circ}$, whereas it is about $-77,7^{\circ}$ in the Dry Valleys.

\subsection{Collaboration with the MIDGE Project}

Tests in Antarctica have been conducted as part of an international collaboration together with the MIDGE (Minimally Invasive Direct Glacial Exploration) project. The goal of this collaboration was to collect a clean brine sample from an Antarctic englacial brine body (e.g. concentrated salt water), located within the Blood Falls crevasse. This location in the McMurdo Dry Valleys, East Antarctica is a unique glacial feature where subglacial liquid is released from a subglacial reservoir approximately $5 \mathrm{~km}$ upstream from the glacier [52] and migrates along the glacier bed prior to its release to the surface. The brine is assumed to be of marine origin $[53,54]$, which is currently further investigated by the US-NSF project MIDGE. Blood Falls is the only known location in Antarctica where significant volumes (thousands of cubic meters per event) of subglacial water are forced to the surface where 
they can be sampled and investigated without deep drilling, making it an ideal terrestrial test site analog to a potential mission scenario to the Tiger Stripes on Enceladus.

\subsection{EnEx Carrier System}

The IceMole worked well both on Morteratsch Glacier as well as in Antarctica. The longest melting channel on Morteratsch Glacier had a length of $25 \mathrm{~m}$. A maximal melting velocity of $1.1 \mathrm{~m} / \mathrm{h}$ and a minimal radius of the melting trajectory of approximately $10 \mathrm{~m}$ could be observed. The different modules of the integrated EnEx system have been tested in scenarios including both horizontal melting, and a starting configuration with an oblique angle of attack. The IceMole could successfully be maneuvered into a horizontal melting path to test its ability to level out from an inclined trajectory. In all of the EnEx field tests the melting scenarios were restricted to one major change in melting direction. This was partially due to time limitations in the field, but also due to minimize the risk of losing the IceMole, as the retraction of the probe through a curved and twisted channel turned out to be a challenging task. The mass and the length of the probe hinders its movements to follow the channel and the probe tends to jam into edges within the ice surface of the melting channel. Integration of the ARS module into the melting head led to a significant decrease of the melting velocity, even after certain system design modifications (compare 2.3). As a consequence the ARS module had to be removed for large parts of the field tests.

In the final field test at Blood Falls, the IceMole successfully penetrated pressurized brine trapped in the Blood Falls crevasse after melting along an inclined, straight melting path of $\approx 17 \mathrm{~m}$ length. In that particular scenario, 


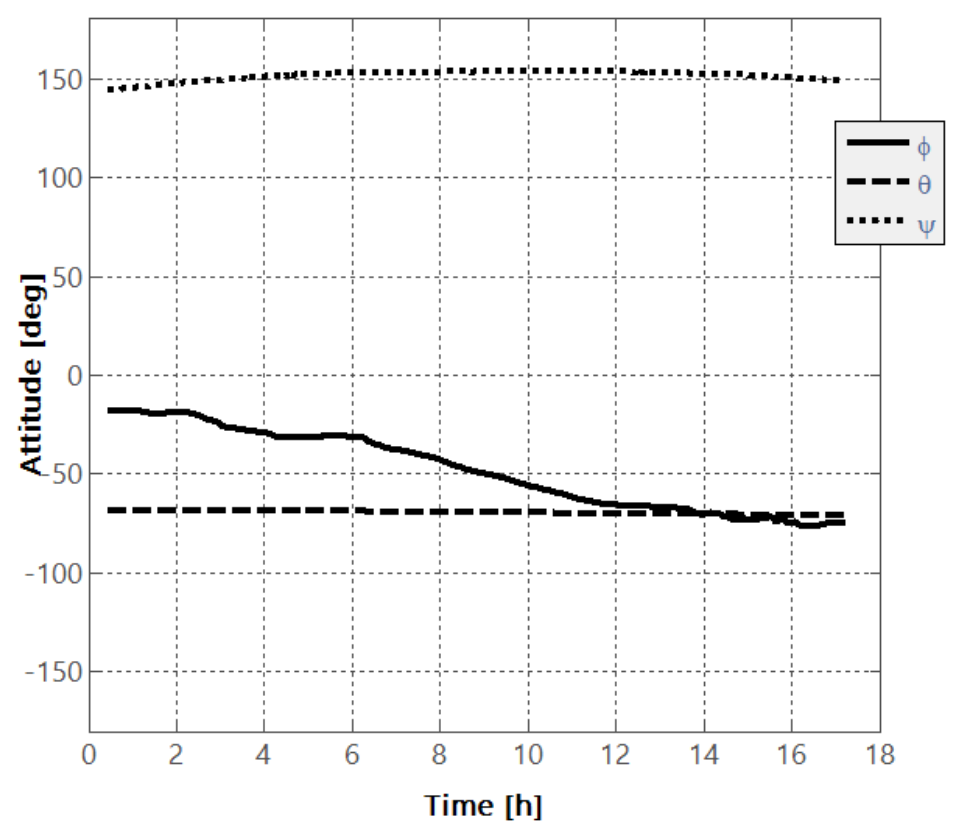

Figure 20: Attitude results after AHRS/MAG fusion for the Blood Falls scenario (17 hours). The roll motion of the probe is clearly visible. It corresponds to a rotation about the main probe axis (roll) and is due to the counter-torque action of the IceMole in response to the rotation of the ice screw. During a forward run the roll delta angle is negative, during the run stops $(4-5 \mathrm{~h})$ the angle stays constant. Positive rotation can be observed at $16.5 \mathrm{~h}$ when unscrewing the ice screw.

there were difficulties with the CASS. However, the IceMole was still able to retract a large volume of subglacial liquid by means of the redundant booster pumping system. Despite difficulties with the CASS during the Blood Falls scenario, the principle functionality of the CASS(penetration of the crevasse, stopping of the IceMole and deployment of the proboscis) has been demonstrated in earlier field tests, including Canada Glacier. 


\subsection{Relative Positioning}

The DR subsystem has been successfully tested at both test sites. Both IMU, and magnetometer could be used at all temperature conditions, even at harsh Antarctic temperatures and high latitudes. The AHRS was able to perform initial alignment, as well as continuous tracking of the IceMoles attitude. The differential magnetometer system was able to stabilize the attitude drift during run and measurement stops. In most of the melting scenarios data of good quality was acquired. Figure 20 shows the computed attitude angles of the probe for the final melting scenario at the Blood Falls.

\subsection{Acoustic Ice Properties}

Dependencies of the speed of sound and acoustic signal attenuation on depth and direction in the glacier have been investigated. The attenuation is important for determining the imaging range of the APS. Information about the behavior of the speed of sound is crucial for the acoustic navigation itself. In order to analyze dependencies on depth and distance, additional acoustic transducers have been inserted in holes of greater depth. Also, different transducer combinations have been analyzed. Horizontal distances between the holes have been determined by differential GPS. The propagation time has been determined by the first crossing of a fixed threshold. The resulting speed of sound shows fluctuations of up to $10 \%$ depending on the depth but constant with time. Other test sites show different depth-profiles but also variations of the same order of magnitude. The speed of sound measured within the transducer array shows a directional variation of $<5 \%$. To develop a predictive model for these effects further studies are necessary. Figure 21 


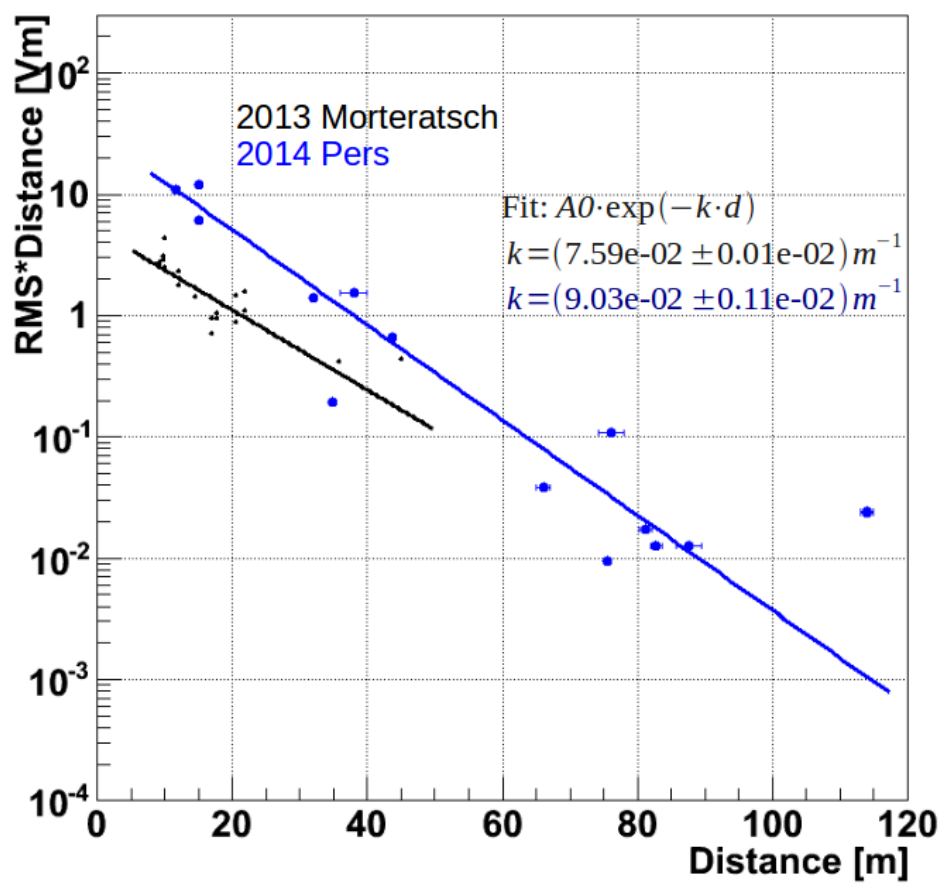

Figure 21: Determination of the attenuation length in glacier ice from the width of averaged acoustic pulses for various distances. The fit is done with the parameter A0 for the amplitude of the exponential function and the parameter $\mathrm{k}$ for the attenuation coefficient.

shows the determination of the attenuation length from the RMS (root-meansquare) of the signals at different distances for the 2013 and 2014 Morteratsch field tests. The determination based on the RMS is more stable than on the amplitude. Losses of acoustic energy due to spherical spreading are taken into account. For these measurements the transducer and the receiver were inserted in different holes in the same depth and the distance of transducer and receiver was varied by using different pairs of holes. From the attenuation 
coefficient of the exponential fit the attenuation length was determined to be $(13.18 \pm 0.02) \mathrm{m}$ in 2013 and $(11.07 \pm 0.12) \mathrm{m}$ in 2014. In 2013 it was possible to receive a signal at a distance of $45 \mathrm{~m}$ with a signal-to-noise ratio sufficient for a precise measurement of the arrival time. This allowed to deduce on the speed of sound, which was determined to be $(3738 \pm 18) \mathrm{m} / \mathrm{s}$. In 2014 a significantly increased acoustic range of $130 \mathrm{~m}$ has been accomplished through improved power dissipation and frequency matching.

\subsubsection{Absolute Positioning}

The feasibility of absolute positioning in glacier ice based upon trilateration of acoustic signals has been demonstrated. Figure 22 shows the reconstruction of a sample straight trajectory. Each circle represents the reconstructed position of the IceMole at the time of the corresponding measurement stop. An accuracy of the positioning of better than $17 \mathrm{~cm}$ could be achieved. This value denotes the median distance from a straight trajectory. A robust signal detection has been accomplished up to an emitter-receiver distance of about $20 \mathrm{~m}$.

\section{Conclusions and Outlook}

\subsection{Conclusions}

In this paper, the EnEx system, consisting of the melting probe IceMole and its navigation system, have been described. The EnEx system integrates an extensive set of sensors like an IMU and magnetometers for attitude determination, an odometer based on ice screw rotation, an acoustic positioning system and an acoustic reconnaissance system. To consolidate all available 


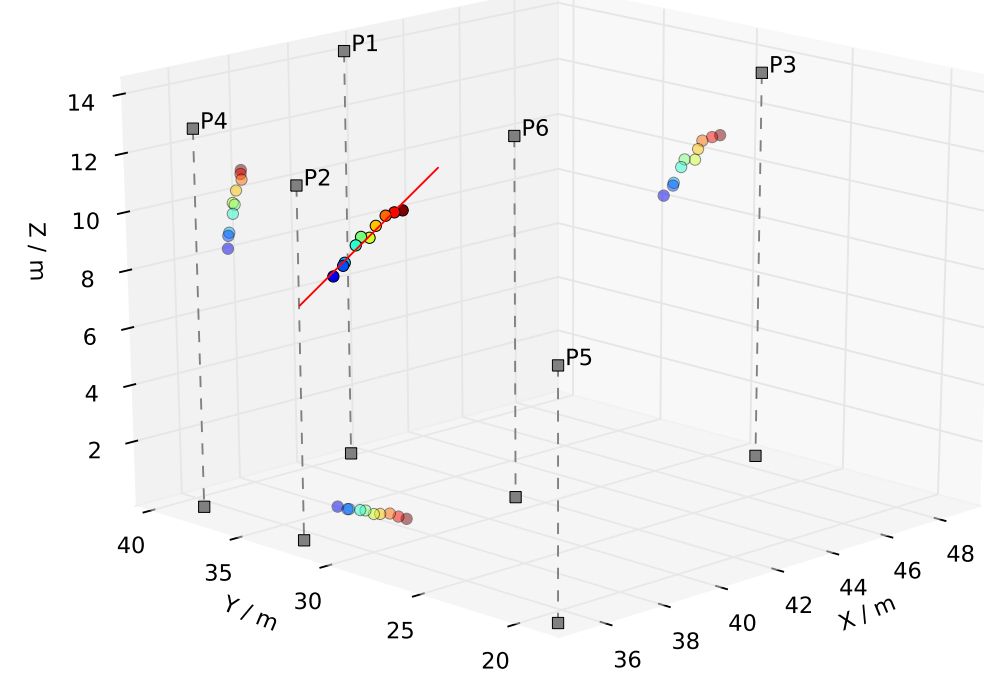

Figure 22: Reconstructed trajectory recorded during a field test on the Morteratsch Glacier in 2014. Each reconstructed position is displayed as a colored circle. The time corresponding to a certain reconstructed position is color coded, where red represents early times and blue represents later times. The trajectory is projected to each coordinate plane for reasons of clarity. The positions of the six emitters as well as their projection on the $\mathrm{x}-\mathrm{y}$ plane are displayed as gray boxes. 
sensor data, a sophisticated multi-sensor fusion based on evidential SLAM has been developed. Functionality and performance of the system have been demonstrated in laboratory and field tests. In particular,

1. the IceMole is capable of melting into temperate and poly-thermal terrestrial glacier ice, both in vertical down direction as well as at an oblique angle. It is capable of changing its melting direction during melting. The integrated pumping system keeps the water column within the channel low. The CASS allows collection of liquid samples. Melting tends to have a lower efficiency as the ambient ice gets colder [55]. This will have to be further studied both experimentally and numerically.

2. DR yields reliable attitude and position data for the slow melting motion through terrestrial glacier ice. Updating the IMU data with magnetic measurements compensates for increasing errors of inertial-only attitude determination and improves accuracy of the results. Differential magnetometry based on a magnetic reference system is suitable for correcting local errors and variations. The working principle relies on the presence of any magnetic field. In the case of Enceladus a strong, homogenous and already well described magnetic field $(\mathbf{B} 0=325 \mathrm{nT})$ is provided by Saturn. As the baseline magnetic field is constantly tracked via the reference station it is not necessary to know the direction of the field a-priori. Hence, equal results in precision and reliability of relative positioning can be expected for Enceladus, after modifying the sensors according to the local environmental conditions.

3. Absolute in-ice positioning based on acoustics is possible, both in temperate and in poly-thermal terrestrial glaciers. The maximal emitter- 
receiver distance up to which a robust signal detection is possible can be further increased by: a larger signal power, a better frequency matching between the acoustic receivers in the IceMole and the ambient ice properties, a better coupling of the emitters to the ice, and an improved signal processing. Though the principle concept is expected to work also at Enceladus conditions, a real systems set-up for a space mission would require additional knowledge on local ice structure and quality.

4. The ARS concept enables forward looking acoustical imaging. Initially, specification of the piezo arrays was based on the physical properties of ice and principles of sonography. The acquired beam forming quality and lateral resolution however was better than expected and actually needed. Findings suggest a redesign of the phased arrays with focus on the resonance frequency and the number of elements to reduce the lateral resolution in favor of range. Although the ARS performance is very promising it turned out to be challenging to integrate the sensors into the melting head while maintaining an acceptable melting efficiency. This difficulty has not been solved yet. It is rather one objective of the ongoing research to reduce the thermally inefficient area by applying copper covers or grids and/or reducing of the number of elements.

5. The multi-sensor fusion combines all available sensor data. It provides an absolute, drift-minimizing position and attitude estimate with higher accuracy than provided by the individual subsystems. Furthermore, a global 3D map is computed from local 2D measurements, while the belief function representation provides an additional dimension of uncertainty useful for operator support and autonomous decision making. 


\subsection{Outlook}

The development of a fully capable ice-penetrating probe for an astrobiological mission to Enceladus or another icy body in the Solar System will be a long, multi-stage process with each phase being aimed at optimizing various aspects of the probe. Within the EnEx - Enceladus Explorer Initiative of the DLR Space Administration this challenging and exciting scientific and technical goal will be further pursued. Crucial to a future mission to Enceladus is the level of autonomy of the system. In any extraterrestrial exploration mission, the one-way signal travel time to Earth is several minutes up to hours, depending on the target body. Therefore, the probe must be able to navigate autonomously through the structurally highly uncertain icy body and be able to respond to target and obstacle detection during run time. This is a challenging task for current technical systems. One solution could be to install an acoustic sensor network on the surface and at an appropriate depth within the ice that enables robust positioning. A difficulty of this idea is the initialization of each acoustic sensor with its exact position in order to relate to that information during the motion of the melting probe. This could be overcome by deploying the acoustic sensors with corner reflectors in which case their coordinates could be determined by laser ranging and imaging from an orbiting satellite [56]. Independent knowledge about the sensors initial locations could then also be used to gain further information on the environment and the ice structure. The icy environment itself, however, is another major source of uncertainty, not only for the applicability of an acoustic sensor network but for all technological components of an Enceladus exploration mission. Few experimental studies address heat conduction, ice 
structure, acoustic wave propagation including scattering and absorption, and generally the nature of water ice metamorphism under very cold ice and low pressure conditions. Around the geologically very active area around Tiger Stripes, Enceladus' surface is covered by precipitated and potentially amorphous ice particles that have previously been ejected into space. Close to the Tiger Stripes, temperatures could be high enough for these particles to bond, which could result in an extreme variability of ice the structure. Both laboratory experiments and ab-initio computer simulations would help to gain a better understanding of very cold icy environments. Further experiments during a preliminary lander mission on either Enceladus or any other of the icy moons in our Solar System could then address the structural uncertainty. The expected large uncertainties in the acquired sensor data requires us to handle uncertain, incomplete and unexpected information. The approach chosen in the project Enceladus Explorer relies on bio-inspired strategies. This approach can be further pursued by developing cognitively motivated strategies for autonomous navigation. Another mandatory requirement fo a future space mission to Enceladus is the miniaturization of the IceMole system to reduce the necessary heating power. A potential mission scenario based on the EnEx-IceMole technology which also discussed power requirements is presented in [5]. Miniaturization will furthermore increase the probe's maneuverability and decrease its mass. Consequently, all components have to be either smaller or replaced by a more light-weight technology with similar functionality. As for the attitude determination, there are miniaturized IMUs based on the so-called MEMS technology, which in principal show a similar level of accuracy and stability, however, have to be strongly supported by 
magnetometer measurements, new evaluation algorithms and/or additional observations. Finally, the melting process itself needs to be further investigated as the environmental conditions on the icy moons of our Solar System imply almost vacuum conditions. This translates into the possibility of a phase change below the triple point, hence, within the sublimation regime, and poses a further challenge. Though there is experimental evidence that sublimation does not play an important role even in vacuum [57], we need to conduct further theoretical and experimental studies to gain a better understanding of the dynamic behavior of melting probes under extraterrestrial conditions.

\section{Contributions}

The EnEx project is based on an idea and initiative of the DLR Space Administration. The EnEx-IceMole carrier system including its sampling system CASS as well as the supporting infrastructure for the field tests, and the simulation model have been developed at the Faculty of Aerospace Engineering, FH Aachen University of Applied Sciences. The DR system has been collaboratively developed by the Institute of Space Technology and Space Applications LRT 9.3, Universität der Bundeswehr München (IMU/AHRS), the Institute of Flight Guidance, Technische Universität Braunschweig (differential magnetometer/attitude update), and the Cognitive Neuroinformatics group at University of Bremen (integration as part of the sensor fusion). The APS has been developed by the III. Physikalisches Institut, RWTH Aachen University and the Department of Physics, Bergische Universität Wuppertal. The ARS has been developed at the Institute of Bioengineering at FH Aachen 
University of Applied Sciences and the III. Physikalisches Institut, RWTH Aachen University. SF was done by the Cognitive Neuroinformatics group at University of Bremen. The concept for a lander mission to Enceladus was studied by the Institute of Space Technology and Space Applications LRT 9.1, Universität der Bundeswehr München. The clean access strategy has been developed collaboratively between the Institute of Bioengineering, FH Aachen University of Applied Sciences and scientists from the MIDGE project. Field tests in Europe have been organized and logistically supported by FH Aachen University of Applied Sciences. Field tests in Antarctica have been collaboratively organized by FH Aachen University of Applied Sciences and partners from the MIDGE project, who provided logistical and financial support through the NSF. Each group contributed to this manuscript by providing a topic specific section. Outlining and editing has been conducted at FH Aachen University of Applied Sciences.

\section{Acknowledgements}

The EnEx project (grants 50NA1206 to 50NA1211) and the EnEx Initiative is funded by DLR Space Administration with financial means of the Federal Ministry of Economic Affairs and Energy on a basis of a decision by the German Bundestag. The MIDGE project is funded by the US National Science Foundation Office of Polar Programs under grants ANT-1144178 and ANT-1144192. 


\section{References}

[1] C. Parkinson, et al., Enceladus: Cassini observations and implications for the search for life, Astronomy and Astrophysics 463 (1) (2007) 353-357.

[2] F. Postberg, et al., A salt-water reservoir as the source of a compositionally stratified plume on Enceladus, Nature 474 (2011) 620-622.

[3] B. Dachwald, et al., Clean In-Situ Subsurface Exploration of Icy Environments in the Solar System, in: J.-P. de Vera, J. Seckbach (Eds.), Habitability of Other Planets and Satellites, Springer, 2013, pp. 367-397.

[4] C. Porco, et al., How the geysers, tidal stresses, and thermal emission across the South Polar Terrain of Enceladus are related, Astron. J. 148 (2014) 45-69.

[5] K. Konstantinidis, et al., A lander mission to probe subglacial water on Saturn's moon Enceladus for life, Acta astronautica Vol. 106 (2014) 63 89.

[6] H.-W. Hsu, et al., Ongoing hydrothermal activities within Enceladus, Nature 519 (2015) 207-211.

[7] J. N. Spitale, et al., Curtain eruptions from enceladus south-polar terrain, Nature 521 (2015) 57-60.

[8] J. Spencer, et al., Enceladus: An active cryovolcanic satellite, in: M. Dougherty, L. Esposito, S. Krimigis (Eds.), Saturn from CassiniHuygens, Springer Netherlands, 2009, pp. 683-724. 
[9] B. Dachwald, et al., Enceladus Explorer - A Maneuverable Subsurface Probe for Autonomous Navigation Through Deep Ice, in: IAC, Naples, Italy, 2012, IAC-12-A3.5.7.

[10] B. Dachwald, et al., IceMole: A maneuverable probe for clean in situ analysis and sampling of subsurface ice and subglacial aquatic ecosystems, Annals of Glaciology 55(65) (2014) 14-22.

[11] H. Aamot, Pendulum steering for thermal probes in glaciers, Journal of Glaciology 6 (1967) 935-939.

[12] H. Aamot, Development of a vertically stabilized thermal probe for studies in and below ice sheets, Journal of Engineering for Industry 92 (2) (1970) 263-268.

[13] M. Moallemi, B. Webb, R. Viskanta, An experimental and analytical study of close-contact melting, Transactions of the ASME 108 (1986) 894-898.

[14] K. Schüller, Development of a finite element model to describe the dynamic behavior of the maneuverable ice exploration probe IceMole (01- 2015).

[15] R. Shreve, Theory of performance of isothermal solid-nose hot-points boring in temperate ice, J. Glaciol 4 (32) (1962) 151-160.

[16] K. Schüller, J. Kowalski, P. Raback, Curvilinear melting - a preliminary experimental and numerical study, Journal of Heat and Mass Transfer (submitted). 
[17] National Research Council (U.S.). Polar Research Board., Exploration of antarctic subglacial aquatic environments : environmental and scientific stewardship, National Academies Press, Washington, D.C., 2007.

[18] H. Niedermeier, et al., Navigation system for a research ice probe for antarctic glaciers, in: PLANS, IEEE/ION, 2014.

[19] H. Hou, N. El-Sheimy, Inertial sensors errors modeling using allan variance, in: ION/GNSS 2003, ION, 2003.

[20] P. Savage, Strapdown Analytics, no. Bd. 1 in Strapdown analytics, Strapdown Associates, 2000.

[21] D. Goshen-Meskin, I. Bar-Itzhack, Unified approach to inertial navigation system error modeling, Journal of Guidance, Contral and Dynamics 15 (1992) 648-653.

[22] J. W. W. Li, Effective Adaptive Kalman Filter for MEMSIMU/Magnetometer Integrated Attitude and Heading Reference Systems, in: Journal of Navigation / Volume 66 / Issue 01, The Royal Institute of Navigation, 2013.

[23] D. Eliseev, et al., Acoustic In-ice Positioning in the Enceladus Explorer Project, Annals of Glaciology.

[24] D. E. Manolakis, Efficient solution and performance analysis of 3-D position estimation by trilateration, Aerospace and Electronic Systems, IEEE Transactions on 32 (4) (1996) 1239-1248. 
[25] I. Malecki, Physical Foundations of Technical Acoustics, Pergamon Press, Oxford, 1969.

[26] C. Vogt, K. Laihem, C. Wiebusch, Speed of sound in bubble-free ice., Journal of the Acoustical Society of America 124 (2008) 3613-3618.

[27] S. Herron, et al., Ultrasonic velocities and crystalline anisotropy in the ice core from Dye 3, Greenland, Geophysical Monograph Series 33 (1985) $23-31$.

[28] A. J. Gow, et al., Physical and structural properties of the Greenland Ice Sheet Project 2 ice core : A review properties paralleled closely those found in cores from Byrd and Die, Journal of Geophysical Research 102 (1997) 26,559-26,575.

[29] A. R., et al., Measurement of sound speed vs. depth in South Pole ice for neutrino astronomy - IceCube Collaboration, Astropart.Phys. 33 (2010) $277-286$.

[30] R. Abbasi, et al., Measurement of Acoustic Attenuation in South Pole Ice, Astropart. Phys. 34 (2011) 382-393.

[31] P. B. Price, Attenuation of acoustic waves in glacial ice and salt domes, Journal of Geophysical Research: Solid Earth 111 (B2) (2006) n/a-n/a, b02201.

[32] R. X. Gao, R. Yan, Wavelets: Theory and Applications for Manufacturing, Springer Verlag, 2010. 
[33] M. Meyer, Signalverarbeitung: Analoge und Digitale Signale, Systeme und Filter, Vieweg+Teubner Verlag, 2011.

[34] C. J. Harvey, et al., Advances in ultrasound., Clinical radiology 57 (3) (2002) 157-77. doi:10.1053/crad.2001.0918.

[35] J. A. Jensen, Medical ultrasound imaging., Progress in biophysics and molecular biology 93 (1-3) (2007) 153-65.

[36] B. W. Drinkwater, P. D. Wilcox, Ultrasonic arrays for non-destructive evaluation: A review, NDT \& E International 39 (7) (2006) 525-541.

[37] J. Habermehl, A. Lamarre, Ultrasonic Phased Array tools for composite inspection during maintenance and manufacturing, in: 17th World Conference on Nondestructive Testing, Shanghai, China, 2008, pp. 25-28.

[38] J. T. Pedicone, et al., Real-time phased array imaging using digital beam forming and autonomous channel control, in: Ultrasonics Symposium, 1990, pp. 1499-1502.

[39] J.-Y. Lu, et al., Biomedical ultrasound beam forming, Ultrasound in Medicine \& Biology 20 (5) (1994) 403-428.

[40] V. A. D. Grosso, C. W. Mader, Speed of Sound in Pure Water, J. Acoust. Soc. Amer.

[41] S. Bencroft, An algebraic solution of the GPS equations, Aerospace and Electronic Systems, IEEE Transactions on AES-21 (1) (1985) 56-59. 
[42] M. George, S. Sukkarieh, Tightly coupled INS/GPS with bias estimation for UAV applications, in: Proceedings of Australiasian Conference on Robotics and Automation (ACRA), 2005.

[43] T. Yang, V. Aitken, Evidential mapping for mobile robots with range sensors, IEEE Transactions on Instrumentation and Measurement 55 (4) (2006) 1422-1429.

[44] J. Mullane, et al., Evidential versus bayesian estimation for radar map building, in: ICARCV'06. 9th International Conference on Control, Automation, Robotics and Vision, IEEE, 2006, pp. 1-8.

[45] M. Ribo, A. Pinz, A comparison of three uncertainty calculi for building sonar-based occupancy grids, Robotics and Autonomous Systems 35 (3-4) (2001) 201-209. doi:10.1016/S0921-8890(01)00116-6.

[46] P. Smets, R. Kennes, The transferable belief model, Artificial Intelligence 66 (1994) 191-234.

[47] T. Reineking, J. Clemens, Dimensions of uncertainty in evidential grid maps, in: C. Freksa, et al. (Eds.), Spatial Cognition IX, Vol. 8684 of Lecture Notes in Computer Science, Springer International Publishing, 2014, pp. 283-298.

[48] J. Clemens, T. Reineking, Multi-Sensor Fusion Using Evidential SLAM for Navigating a Probe through Deep Ice, in: F. Cuzzolin (Ed.), Belief Functions: Theory and Applications, Vol. 8764 of Lecture Notes in Computer Science, Springer International Publishing, 2014, pp. 339-347. doi:10.1007/978-3-319-11191-9_37. 
[49] H. Durrant-Whyte, T. Bailey, Simultaneous localization and mapping: part i, IEEE Robotics \& Automation Magazine 13 (2) (2006) 99-110.

[50] M. Montemerlo, et al., FastSLAM: A factored solution to the simultaneous localization and mapping problem, in: Proceedings of the National conference on Artificial Intelligence, 2002, pp. 593-598.

[51] T. Reineking, J. Clemens, Evidential FastSLAM for grid mapping, in: 16th International Conference on Information Fusion (FUSION), 2013, pp. 789-796.

[52] A. Hubbard, et al., Evidence for subglacial ponding across Taylor Glacier, Dry Valleys, Antarctica, Ann. Glaciol. 39 (2004) 79-84.

[53] J. Mikucki, et al., Geomicrobiology of Blood Falls: An iron-rich saline discharge at the terminus of the Taylor Glacier, Antarctica, Aquatic Geochemistry 10 (2004) 199-220.

[54] D. Pollard, R. DeConto, Modeling West Antarctic ice sheet growth and collapse through the past five million years, Nature 458 (2009) 329-332.

[55] S. Ulamec, et al., Access to Glacial and Subglacial Environments in the Solar System by Melting Probe Technology, Reviews in Environmental Science and Biotechnology 6 (1) (2007) 71-94.

[56] C. Gisinger, et al., Precise Three-Dimensional Stereo Localization of Corner Reflectors and Persistent Scatterers With TerraSAR-X, IEEE Trans. on Geoscience and Remote Sensing 53 (4) (2015) 1782-1802. 
1109 [57] J. Biele, et al., In situ analysis of Europa ices by short-range melting $1110 \quad$ probes, Advances in Space Research 48 (4) (2011) 755-763. 


\section{Graphical Abstract:}

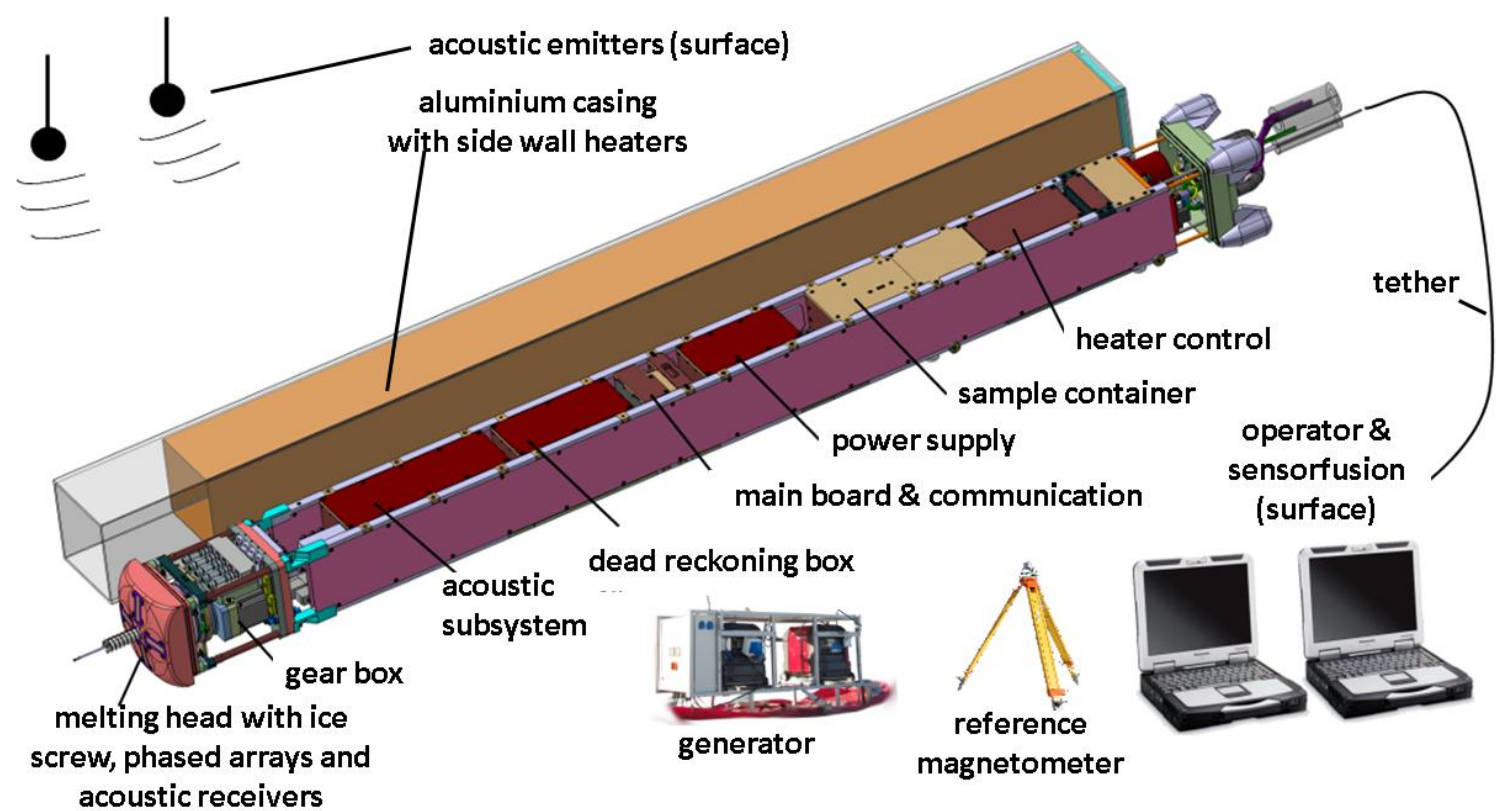

